\title{
Agravity up to infinite energy
}

\author{
Alberto Salvio $^{1, \mathrm{a}}$, Alessandro Strumia ${ }^{2}$ \\ ${ }^{1}$ Theoretical Physics Department, CERN, Geneva, Switzerland \\ 2 Dipartimento di Fisica dell'Universitá di Pisa and INFN, Pisa, Italy
}

Received: 7 July 2017 / Accepted: 26 January 2018 / Published online: 10 February 2018

(C) The Author(s) 2018. This article is an open access publication

\begin{abstract}
The self-interactions of the conformal mode of the graviton are controlled, in dimensionless gravity theories (agravity), by a coupling $f_{0}$ that is not asymptotically free. We show that, nevertheless, agravity can be a complete theory valid up to infinite energy. When $f_{0}$ grows to large values, the conformal mode of the graviton decouples from the rest of the theory and does not hit any Landau pole provided that scalars are asymptotically conformally coupled and all other couplings approach fixed points. Then agravity can flow to conformal gravity at infinite energy. We identify scenarios where the Higgs mass does not receive unnaturally large physical corrections. We also show a useful equivalence between agravity and conformal gravity plus two extra conformally coupled scalars, and we give a simpler form for the renormalization group equations of dimensionless couplings as well as of massive parameters in the presence of the most general matter sector.
\end{abstract}

\section{Contents}

1 Introduction $\ldots \ldots \ldots \ldots \ldots \ldots \ldots$

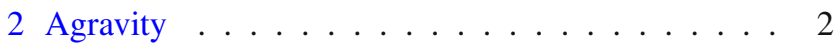

3 Conformal gravity . . . . . . . . . . 5

4 The conformal mode of the agraviton . . . . . 7

5 Scenarios compatible with naturalness of the Higgs mass ...................... 9

6 Conclusions . . . . . . . . . . . . 11

A One-loop RGE in agravity . . . . . . . . . . . . 12

B One-loop RGE for massive parameters in agravity . 13

References . . . . . . . . . . . . . . . 13

\section{Introduction}

The idea that scalars, like the Higgs, must be accompanied by new physics that protects their lightness from power-

\footnotetext{
a e-mail: alberto.salvio@cern.ch
}

divergent quantum corrections led to the following view of mass scales in nature: the weak scale is the supersymmetric scale, and the Planck scale is the string scale. The nonobservation of supersymmetric particles around the weak scale challenged this scenario, leading to the alternative idea that only physical corrections to scalar masses must satisfy naturalness. Namely, extra new particles with mass $M_{\text {extra }}$ and coupling $g_{\text {extra }}$ to the Higgs, must satisfy

$\delta M_{h} \sim g_{\text {extra }} M_{\text {extra }} \lesssim M_{h}$.

A rationale for ignoring power-divergent corrections is the following. The one-loop quantum correction to the masses of scalars, vectors and of the graviton is power divergent, showing the dangers of attributing physical meaning to powerdivergent corrections. A cut-off (such as string theory) that knows that vector and graviton masses are protected by gauge invariance can keep them to zero, while giving a large correction to scalar masses. A less smart cut-off (such as dimensional regularization) can be blind to the difference, and set to zero all power divergences. The simplest cut-off with this property is no cut-off: a theory where all renormalizable couplings flow up to infinite energy without hitting Landau poles.

The above arguments motivate the following scenario: if nature is described at fundamental level by a dimensionless Lagrangian, all power-divergent quantum correctionsbeing dimensionful-must be interpreted as vanishing. Taking gravity into account, the most general dimensionless action in $3+1$ space-time dimensions contains gauge couplings, Yukawa couplings, scalar quartics, non-minimal $\xi$ couplings between scalars and gravity and, in the purely gravitational sector, two dimensionless gravitational couplings, $f_{0}$ and $f_{2}$, analogous to gauge couplings:

$$
S=\int \mathrm{d}^{4} x \sqrt{|\operatorname{det} g|}\left[\frac{R^{2}}{6 f_{0}^{2}}+\frac{\frac{1}{3} R^{2}-R_{\mu \nu}^{2}}{f_{2}^{2}}+\mathscr{L}_{\text {matter }}\right],
$$

where $\mathscr{L}_{\text {matter }}$ corresponds to the part of the Lagrangian that depends on the matter fields, with dimensionless parameters only. This theory [1] is renormalizable, as suggested in [2] 
and formally proven in [3]. The weak scale, the QCD scale and the Planck scale can be dynamically generated [4] from vacuum expectation values or from condensates. Perturbative dimensionless theories automatically give slow-roll inflation [4-9] (see also refs. [10,11] for related studies).

However, Eq. (2) means that four derivatives act on the graviton: thereby some graviton components have a negative kinetic term. ${ }^{1}$ Classically the theory in (2) is sick [13]: the energy is unbounded from below. A sensible quantum theory might exist, analogously to what happens with fermions: their classical energy is negative, but their quantum theory is sensible. ${ }^{2}$ We will not address this problem here.

We will here study whether this theory can flow up to infinite energy. The Quantum Field Theory (QFT) part can have this property. Realistic TeV-scale extensions of the Standard Model (SM) can be asymptotically free [23,24], and it is not known whether the SM itself can be asymptotically safe, in a non-perturbative regime [25]. The gravitational coupling $f_{2}$ is asymptotically free. The difficulty resides in the coupling $f_{0}$ : a small $f_{0}$ grows with energy, until it becomes large.

In this paper we will show that, despite this, the theory can flow up to infinite energy, in an unusual way. In Sect. 2 we present an alternative formulation of agravity that makes it easier to compute its renormalization group equations (RGE): $f_{0}$ becomes the quartic of a special scalar, the conformal mode of the agraviton. Then a large $f_{0}$ means that the conformal mode of the agraviton gets strongly selfcoupled. The rest of the theory decouples from it, if at the same time all scalars become conformally coupled, namely if all $\xi$ parameters run to $-1 / 6$, and all the other couplings reach ultraviolet (UV) fixed points, where all $\beta$-functions vanish.

In Sect. 4 we isolate the conformal mode of the graviton and show that its strong dynamics is such that $f_{0}$ does not hit a Landau pole. This means that the infinite-energy limit of agravity can be conformal gravity. The unusual phenomenon that allows one to reach infinite energy is that the conformal mode of the graviton fluctuates freely, but the rest of theory is not coupled to it: it becomes a gauge redundancy of a new local symmetry, Weyl symmetry. Since this symmetry is anomalous, conformal gravity cannot be the complete theory: going to lower energy the conformal model of the graviton

\footnotetext{
1 This can maybe be avoided introducing an infinite series of higher derivative terms [12], but the resulting gravity theories contain infinite free parameters and are not known to be renormalizable.

2 The ample literature of 'ghosts' was critically reviewed in [14]; for later work, see [15-22], where it was proposed that a four-derivative variable $q(t)$ contains two canonical degrees of freedom (d.o.f.), $q_{1}=$ $q$ and $q_{2}=\dot{q}$, with opposite time-reflection parity, such that usual $T$-even representation $\left(q_{1}|x\rangle=x|x\rangle\right.$ and $\left.p_{1}|x\rangle=i \frac{\mathrm{d}}{\mathrm{d} x}|x\rangle\right)$ must be combined with the $T$-odd representation $\left(q_{2}|y\rangle=i y|y\rangle\right.$ and $p_{2}|y\rangle=$ $\frac{\mathrm{d}}{\mathrm{d} y}|y\rangle$ ) obtaining consistent results (positive energy, normalizable wave functions, Euclidean continuation), although the interpretation of the resulting negative norm is unclear.
}

starts coupling to the rest of the theory, which becomes agravity. This issue is discussed in Sect. 3. In Sect. 5 we propose scenarios where the Higgs mass does not receive unnaturally large corrections. Conclusions are given in Sect. 6. Finally, in the appendix we provide a new and simple expression for the one-loop RGE of all dimensionless parameters (Appendix A) as well as of all dimensionful parameters (Appendix B) in the presence of the most general matter sector, which was not studied before.

\section{Agravity}

Allowing for generic scalars $\phi_{a}$ with generic dimensionless coupling $\xi_{a b}$ to gravity, $-\frac{1}{2} \xi_{a b} \phi_{a} \phi_{b} R$, the one-loop RGE for $f_{0}$ is $[4,26-28]$

$$
\begin{aligned}
(4 \pi)^{2} \frac{\mathrm{d} f_{0}^{2}}{\mathrm{~d} \ln \bar{\mu}}= & \frac{5}{3} f_{2}^{4}+5 f_{2}^{2} f_{0}^{2}+\frac{5}{6} f_{0}^{4} \\
& +\frac{f_{0}^{4}}{12}\left(\delta_{a b}+6 \xi_{a b}\right)\left(\delta_{a b}+6 \xi_{a b}\right)>0 \\
& \quad \text { for } f_{0} \ll 1,
\end{aligned}
$$

where $\bar{\mu}$ is the renormalization scale in the modified minimal subtraction scheme (see also $[29,30]$ for a previous attempt to determine this RGE). This shows that, in all theories, $f_{0}$ is asymptotically free only for $f_{0}^{2}<0$. However, negative $f_{0}^{2}$ corresponds to a run-away potential $[4,6,7]$, and this instability cannot be made harmless (or even beneficial for explaining dark energy) by invoking a small enough negative $f_{0}^{2}$, since tests of gravity exclude extra graviton components below $0.05 \mathrm{eV}$ (see [31,32] for attempts to have $f_{0}^{2}<0$ ). The fact that $f_{0}^{2}<0$ is phenomenologically problematic was already noted in [4], where it was pointed out that it leads to a tachyonic instability. Barring stabilization through background effects in cosmology, one needs $f_{0}^{2}>0$. But the one-loop RGE show that a small $f_{0}^{2}>0$ grows until it becomes non-perturbative. ${ }^{3}$

These RGE show peculiar features. Only scalars (not vectors nor fermions) generate $f_{0}$ at one-loop, and only if their $\xi$-couplings have a non-conformal value, $\xi_{a b} \neq-\delta_{a b} / 6$. The $\xi$-couplings often appear in the RGE in the combination $\xi_{a b}+\delta_{a b} / 6$, but not always. The coupling $f_{0}$ appears at the denominator in the RGE for the $\xi$-couplings [4].

The above features can be understood noticing that a new symmetry appears in the limit $f_{0} \rightarrow \infty$ and $\xi_{a b} \rightarrow-\delta_{a b} / 6$ :

\footnotetext{
${ }^{3}$ Different statements in the literature (even recent) appear either because some previous results contained wrong signs or because some authors use computational techniques that try to give a physical meaning to power divergences, obtaining gauge-dependent and cut-offdependent results. Claims that a run-away potential with very small $f_{0}$ can mimic Dark Energy do not take into account bounds on extra graviton components.
} 
Table 1 Transformations of coordinates and fields under a Weyl transformation

\begin{tabular}{|c|c|c|c|c|c|c|}
\hline & & Dilatation & $\otimes$ & Diffeomorphism & $=$ & Weyl transformation \\
\hline Coordinates & $\mathrm{d} x^{\mu}$ & $e^{\sigma} \mathrm{d} x^{\mu}$ & & $e^{-\sigma} \mathrm{d} x^{\mu}$ & & $\mathrm{d} x^{\mu}$ \\
\hline Graviton & $g_{\mu \nu}$ & $g_{\mu \nu}$ & & $e^{2 \sigma} g_{\mu \nu}$ & & $e^{2 \sigma} g_{\mu \nu}$ \\
\hline Scalars & $\phi$ & $e^{-\sigma} \phi$ & & $\phi$ & & $e^{-\sigma} \phi$ \\
\hline Vectors & $V_{\mu}$ & $e^{-\sigma} V_{\mu}$ & & $e^{\sigma} V_{\mu}$ & & $V_{\mu}$ \\
\hline Fermions & $\psi$ & $e^{-3 \sigma / 2} \psi$ & & $\psi$ & & $e^{-3 \sigma / 2} \psi$ \\
\hline
\end{tabular}

the Weyl (or local conformal) symmetry. The Weyl symmetry is a local dilatation $\mathrm{d} x^{\mu} \rightarrow e^{\sigma(x)} \mathrm{d} x^{\mu}$ compensated by the special diffeomorphism $\mathrm{d} x^{\mu} \rightarrow e^{-\sigma(x)} \mathrm{d} x^{\mu}$ such that the coordinates $\mathrm{d} x^{\mu}$ remain unaffected. The various fields rescale under a dilatation as determined by their mass dimension, and they transform under a diffeomorphism as dictated by their Lorentz indices, as summarized in Table 1.

Agravity is invariant under global Weyl transformations: being dimensionless, it is invariant under global dilatations (for which $\sigma$ does not depend on $x$ ); being covariant, it is invariant under local diffeomorphisms.

Agravity is not invariant under local Weyl transformations. A generic dimensionless theory can be written in terms of the metric $g_{\mu \nu}$, real scalars $\phi_{a}$, Weyl fermions $\psi_{j}$ and vectors $V_{A}$ (with field strength $F_{\mu \nu}^{A}$ ). The action $S=\int \mathrm{d}^{4} x \sqrt{|\operatorname{det} g|} \mathscr{L}$ can be split as $S=S_{\text {Weyl }}+S_{\text {WeyI }}$ where the first part is invariant under Weyl transformations,

$$
\begin{aligned}
\mathscr{L}_{\text {Weyl }}= & \frac{\frac{1}{3} R^{2}-R_{\mu \nu}^{2}}{f_{2}^{2}}-\frac{1}{4}\left(F_{\mu \nu}^{A}\right)^{2} \\
& +\frac{\left(D_{\mu} \phi_{a}\right)^{2}}{2}+\bar{\psi}_{j} i \not D \psi_{j} \\
& +\frac{1}{12} \phi_{a}^{2} R-\frac{1}{2}\left(Y_{i j}^{a} \psi_{i} \psi_{j} \phi_{a}+\text { h.c. }\right) \\
& -\frac{\lambda_{a b c d}}{4 !} \phi_{a} \phi_{b} \phi_{c} \phi_{d},
\end{aligned}
$$

and the second part,

$$
\begin{aligned}
\mathscr{L}_{\text {WeyT }}= & \frac{R^{2}}{6 f_{0}^{2}}-\frac{1}{2} \zeta_{a b} \phi_{a} \phi_{b} R, \\
& \text { where } \zeta_{a b} \equiv \xi_{a b}+\delta_{a b} / 6,
\end{aligned}
$$

is not invariant. ${ }^{4}$ To see this we will now perform a Weyl transformation,

$$
\begin{aligned}
& g_{\mu \nu}(x) \rightarrow e^{2 \sigma(x)} g_{\mu \nu}(x), \quad \phi(x) \rightarrow e^{-\sigma(x)} \phi(x), \\
& \psi(x) \rightarrow e^{-3 \sigma(x) / 2} \psi(x), \quad V_{\mu} \rightarrow V_{\mu} .
\end{aligned}
$$

This will also lead to an equivalent formulation of the theory.

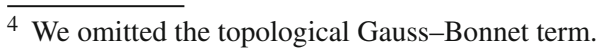

Equivalent formulations of agravity

The extra scalar field $\sigma(x)$, defined in (6), will be called the 'conformal mode of the agraviton'; for the moment it is introduced as an extra gauge redundancy. We will comment on the corresponding gauge symmetry later on.

All terms in Eq. (4) are invariant under Weyl transformations. Since vectors and fermions appear only in Eq. (4), $\sigma$ does not couple to them. Only the terms that break Weyl symmetry give rise to interactions of $\sigma$. The transformation (6) leads to

$$
\begin{aligned}
& \sqrt{|\operatorname{det} g|} \rightarrow e^{4 \sigma} \sqrt{|\operatorname{det} g|}, \\
& R \rightarrow e^{-2 \sigma}\left(R-6 e^{-\sigma} \square e^{\sigma}\right) .
\end{aligned}
$$

Therefore, the Weyl-breaking part of the Lagrangian becomes

$$
\begin{aligned}
& \sqrt{|\operatorname{det} g|} \mathscr{L}_{\text {WeyT }}=\sqrt{|\operatorname{det} g|} \\
& \times\left[\frac{\left(R-6 e^{-\sigma} \square e^{\sigma}\right)^{2}}{6 f_{0}^{2}}-\frac{1}{2} \zeta_{a b} \phi_{a} \phi_{b}\left(R-6 e^{-\sigma} \square e^{\sigma}\right)\right],
\end{aligned}
$$

which is one simple way to rewrite $\mathscr{L}_{\text {Weyt }}$, which will be used later on.

Another simple and useful form of $\mathscr{L}_{\text {WeyT }}$ can be obtained from (8) as follows. We define $\Omega_{L}=e^{\sigma}$ and complete the square rewriting Eq. (8) as

$$
\begin{aligned}
\mathscr{L}_{\text {WeyT }} & =\frac{A^{2}}{6 f_{0}^{2}}-\frac{3}{8} f_{0}^{2}\left(\zeta_{a b} \phi_{a} \phi_{b}\right)^{2}, \\
A & =R-6 \frac{\square \Omega_{L}}{\Omega_{L}}-\frac{3}{2} f_{0}^{2} \zeta_{a b} \phi_{a} \phi_{b} .
\end{aligned}
$$

Next we write the square as $A^{2} / 6 f_{0}^{2}=-\frac{1}{6} f_{0}^{2} \Omega_{L}^{2} \Omega_{R}^{2}+$ $\frac{1}{3} \Omega_{R} \Omega_{L} A$ by introducing an auxiliary field $\Omega_{R}$ with quadratic action, such that integrating it out gives back the original action. The resulting expression only contains the combination $\Omega_{L} \Omega_{R}$, that is, invariant under $\Omega_{L} \rightarrow t \Omega_{L}, \Omega_{R} \rightarrow$ $\Omega_{R} / t$, which forms a $\mathrm{SO}(1,1)$ scale symmetry. Indeed, one can verify that $\mathrm{SO}(1,1)$ is broken by adding Lagrangian terms with dimensionful coefficients, such as the EinsteinHilbert term or the cosmological constant, as done later in Eq. (40). Now, we can rewrite $\Omega_{L} \Omega_{R}$ in vectorial notation as $\Omega_{L} \Omega_{R}=\frac{1}{4}\left(\Omega_{+}^{2}-\Omega_{-}^{2}\right)=\frac{1}{4} \vec{\Omega}^{2}$ by going from the "lightcone basis" $\Omega_{L, R}$ to the $\Omega_{ \pm}$basis as $\Omega_{L}=t\left(\Omega_{+}-\Omega_{-}\right) / 2$ 
and $\Omega_{R}=\left(\Omega_{+}+\Omega_{-}\right) / 2 t$ and defining the $\mathrm{SO}(1,1)$ vector $\vec{\Omega}=\left(\Omega_{+}, \Omega_{-}\right)$. Then the Weyl-breaking part of the action can be written in the final form

$$
\begin{aligned}
& S_{\text {WeyT }}=\int \mathrm{d}^{4} x \sqrt{|\operatorname{det} g|} \\
& \quad \times\left[\frac{g^{\mu \nu}}{2}\left(\partial_{\mu} \vec{\Omega}\right)\left(\partial_{\nu} \vec{\Omega}\right)+\frac{1}{12} \vec{\Omega}^{2} R-\frac{f_{0}^{2}}{96}\left(\vec{\Omega}^{2}+6 \zeta_{a b} \phi_{a} \phi_{b}\right)^{2}\right] .
\end{aligned}
$$

The non-trivial result is that the Weyl-breaking part of the action has been rewritten as an extra Weyl-invariant action involving the extra scalar $\operatorname{SO}(1,1)$ doublet $\vec{\Omega}$, which describes the conformal mode of the agraviton.

We have not (yet) imposed any constraint on the metric $g_{\mu \nu}$ after the transformation in Eq. (6); therefore we have a Weyl-type gauge invariance acting as

$$
\begin{aligned}
& g_{\mu \nu}(x) \rightarrow e^{-2 \chi(x)} g_{\mu \nu}(x), \quad \phi(x) \rightarrow e^{\chi(x)} \phi(x), \\
& \psi(x) \rightarrow e^{3 \chi(x) / 2} \psi(x), \quad V_{\mu} \rightarrow V_{\mu}
\end{aligned}
$$

where $\chi(x)$ is an arbitrary real function of $x$. The transformation $\sigma \rightarrow \sigma+\chi$ is equivalent to including $\Omega_{L}=e^{\sigma}$ and $\Omega_{R}$ among the scalars $\phi$. Therefore, agravity is equivalent to conformal gravity plus two extra conformally coupled scalars, $\Omega_{+}$and $\Omega_{-}{ }^{5}$ In the new formulation of agravity with the field $\vec{\Omega}$, the gravitational couplings $f_{0}$ and $\xi_{a b}$ have become scalar quartic couplings.

The formulations presented in this section certainly are equivalent at the classical level. At quantum level, the equivalence needs to take into account the anomalous transformation law of the path-integral measure, which amounts to adding an effective $\sigma$-dependent term in the action. This amounts to $\sigma$ starting to couple to terms that break scale invariance proportionally to their quantum $\beta$-functions. These extra couplings only affect RGE at higher loop orders, as we will discuss in Sect. 3 .

It is now clear why the one-loop RGE for $f_{0}$, Eq. (3), does not receive contributions from fermions and vectors: $f_{0}^{2}$ is the quartic coupling of a neutral scalar with no Yukawa interactions. A positive $f_{0}^{2}$ corresponds to a positive quartic. Furthermore the symmetry $\mathrm{SO}(1,1)$ can be complexified into $\mathrm{SO}(2)$ by redefining $\Omega_{-} \rightarrow i \Omega_{-}$without affecting the RGE at perturbative level: only non-perturbative large field fluctuations are sensitive to the difference. By defining an extended set of quartic couplings, $\lambda_{\mathrm{ABCD}}$, where the capital indices run such that the quartics that involve the two extra scalars $\vec{\Omega}$ are

\footnotetext{
5 Similar remarks have been made in the context of Einstein gravity (rather than in agravity) in [33-35], where it was found that Einstein gravity is equivalent to conformal gravity plus a single conformally coupled scalar. Similar statements have been made in a different theory without the $R^{2} / 6 f_{0}^{2}$ term in $[36,37]$.
}

included, the generic RGE for the scalar quartics only, known in a generic QFT up to two loops, are ${ }^{6}$

$$
\begin{aligned}
& \frac{\mathrm{d} \lambda_{A B C D}}{\mathrm{~d} \ln \bar{\mu}}=\frac{1}{(4 \pi)^{2}} \sum_{\text {perms }} \frac{1}{8} \lambda_{A B E F} \lambda_{E F C D} \\
& +\frac{1}{(4 \pi)^{4}}\left[\frac{\gamma}{2} \lambda_{A B C D}-\frac{1}{4} \sum_{\text {perms }} \lambda_{A B E F} \lambda_{C E G H} \lambda_{D F G H}\right]+\cdots,
\end{aligned}
$$

where $\gamma=\Lambda_{A A}+\Lambda_{B B}+\Lambda_{C C}+\Lambda_{D D}$ (with $\Lambda_{A B}=$ $\left.\frac{1}{6} \lambda_{A C D E} \lambda_{B C D E}\right)$ is the scalar wave-function renormalization, the sums run over the 4 ! permutations of $A B C D$ and $\cdots$ is the contribution of the other couplings.

From Eq. (12) one can re-derive the one-loop RGE for $f_{0}$ and $\xi_{a b}$, computed as gravitational couplings in [4]. The two results agree. Furthermore, the same RGE acquire a simpler form if rewritten in terms of the $\lambda_{A B C D}$ coefficients. The RGE are explicitly written in Eq. (50) in Appendix A, and neither $f_{0}$ nor any other coupling appear anymore at the denominator in the RGE.

The graviton propagator

A gravitational computation is now only needed to compute the part of the RGE involving $f_{2}$. So far the field $\sigma$, or $\vec{\Omega}$, has been introduced as an extra gauge redundancy. One can fix it by setting $\sigma=0$, going back to the original formulation where the full RGE were computed in [4]. In the rest of this section (which contains technical details used only for a double check of the main results) we show how one can choose an alternative convenient condition: that the fluctuation $h_{\mu \nu}^{\prime}$ around the flat space of $g_{\mu \nu}$ after the transformation in Eq. (6) has vanishing trace, that is,

$h^{\prime} \equiv \eta^{\mu \nu} h_{\mu \nu}^{\prime}=0$.

We have introduced a prime in $h_{\mu \nu}^{\prime}$ to distinguish it from the fluctuation $h_{\mu \nu}$ around the flat space of the metric before transformation (6). The new variables $h_{\mu \nu}^{\prime}$ and $\sigma$ are given in terms of the old ones (the trace $h \equiv \eta^{\mu \nu} h_{\mu \nu}$ and the traceless part $\left.h_{\mu \nu}^{\mathrm{TL}} \equiv h_{\mu \nu}-\eta_{\mu \nu} h / 4\right)$ by

$e^{2 \sigma}=1+\frac{h}{4}, \quad h_{\mu \nu}^{\prime}=e^{-2 \sigma} h_{\mu \nu}^{\mathrm{TL}}$.

The path-integral measure $D g_{\mu \nu} \equiv D h D h_{\mu \nu}^{\mathrm{TL}}$ splits as $D g_{\mu \nu}=D h_{\mu \nu}^{\prime} D \sigma=D h_{\mu \nu}^{\prime} D \vec{\Omega}$. We neglect here the Weyl anomaly because, as explained above, it does not affect the one-loop RGE.

In order to compute quantum effects, we consider the following convenient gauge fixing for the diffeomorphisms $x^{\mu} \rightarrow x^{\mu}+\xi^{\mu}(x)$ :

\footnotetext{
${ }^{6}$ For a recent summary see [38].
} 
$\partial^{\mu} h_{\mu \nu}^{\prime}=0$,

where we use the flat metric $\eta_{\mu \nu}$ to raise and lower the indices. This choice avoids kinetic mixing between $\sigma$ and $h_{\mu \nu}^{\prime}$ and leads to a simple propagator of $h_{\mu \nu}^{\prime}$

$D_{\mu \nu \rho \sigma}^{\prime}=-2 f_{2}^{2} \frac{i}{k^{4}} P_{\mu \nu \rho \sigma}^{(2)}$,

where

$P_{\mu \nu \rho \sigma}^{(2)}=\frac{1}{2} T_{\mu \rho} T_{\nu \sigma}+\frac{1}{2} T_{\mu \sigma} T_{\nu \rho}-\frac{1}{3} T_{\mu \nu} T_{\rho \sigma}$,

$T_{\mu \nu}=\eta_{\mu \nu}-k_{\mu} k_{\nu} / k^{2}$.

To determine the Lagrangian of the Fadeev-Popov ghosts we have to perform the variation of $\partial^{\mu} h_{\mu \nu}^{\prime}$ with respect to diffeomorphisms, whose effect on $h_{\mu \nu}$ at the linear level in $\xi^{\mu}$ is

$h_{\mu \nu} \rightarrow h_{\mu \nu}-\left(\partial_{\mu} \xi_{\nu}+\partial_{\nu} \xi_{\mu}\right)$

$-\left(h_{\alpha \mu} \partial_{\nu}+h_{\alpha \nu} \partial_{\mu}+\left(\partial_{\alpha} h_{\mu \nu}\right)\right) \xi^{\alpha}$.

The effect of diffeomorphisms on $h_{\mu \nu}^{\prime}$ and $\sigma$ can be computed by first splitting Eq. (18) in its traceless and trace parts,

$$
\begin{aligned}
h & \rightarrow h-2 \partial_{\mu} \xi^{\mu}-2 h_{\alpha \mu}^{\mathrm{TL}} \partial^{\mu} \xi^{\alpha}-\frac{1}{2} h \partial_{\mu} \xi^{\mu}-\xi^{\alpha} \partial_{\alpha} h, \\
h_{\mu \nu}^{\mathrm{TL}} & \rightarrow h_{\mu \nu}^{\mathrm{TL}}-\partial_{\nu} \xi_{\mu}-\partial_{\mu} \xi_{\nu}+\frac{1}{2} \eta_{\mu \nu} \partial_{\alpha} \xi^{\alpha} \\
& -h_{\alpha \mu}^{\mathrm{TL}} \partial_{\nu} \xi^{\alpha}-h_{\alpha \nu}^{\mathrm{TL}} \partial_{\mu} \xi^{\alpha}-\partial_{\alpha} h_{\mu \nu}^{\mathrm{TL}} \xi^{\alpha} \\
& +\frac{1}{2} \eta_{\mu \nu} h_{\alpha \beta}^{\mathrm{TL}} \partial^{\beta} \xi^{\alpha}-\frac{1}{4} h\left(\partial_{\nu} \xi_{\mu}+\partial_{\mu} \xi_{\nu}\right) \\
& +\frac{1}{8} \eta_{\mu \nu} h \partial_{\alpha} \xi^{\alpha},
\end{aligned}
$$

and next by using Eq. (14) to express $h_{\mu \nu}^{\mathrm{TL}}$ and $h$ in terms of $h_{\mu \nu}^{\prime}$ and $\sigma$. The result is

$$
\begin{aligned}
e^{2 \sigma} \rightarrow & e^{2 \sigma}\left(1-\frac{1}{2} \partial_{\mu} \xi^{\mu}-\frac{1}{2} h_{\mu \alpha}^{\prime} \partial^{\mu} \xi^{\alpha}-2 \xi_{\alpha} \partial^{\alpha} \sigma\right) \\
h_{\mu \nu}^{\prime} \rightarrow & h_{\mu \nu}^{\prime}-\partial_{\nu} \xi_{\mu}-\partial_{\mu} \xi_{\nu}+\frac{1}{2} \eta_{\mu \nu} \partial_{\alpha} \xi^{\alpha} \\
& -h_{\alpha \mu}^{\prime} \partial_{\nu} \xi^{\alpha}-h_{\alpha \nu}^{\prime} \partial_{\mu} \xi^{\alpha}-\partial_{\alpha} h_{\mu \nu}^{\prime} \xi^{\alpha}+\frac{1}{2} h_{\mu \nu}^{\prime} \partial_{\alpha} \xi^{\alpha} \\
& +\frac{1}{2} \eta_{\mu \nu} h_{\alpha \beta}^{\prime} \partial^{\beta} \xi^{\alpha}+\frac{1}{2} h_{\mu \nu}^{\prime} h_{\alpha \beta}^{\prime} \partial^{\beta} \xi^{\alpha} .
\end{aligned}
$$

Notice that the transformation law of $h_{\mu \nu}^{\prime}$ is independent of $\sigma$ : having used the gauge in Eq. (15) the Fadeev-Popov procedure does not generate any new coupling of $\sigma$ to the Fadeev-Popov ghosts. ${ }^{7}$ In conclusion, we have shown how to implement the gauge where the graviton is traceless.

\footnotetext{
${ }^{7}$ We treated the Weyl transformation as a change of variables in field space. We could equivalently have seen it as an extra gauge redundancy. In this alternative formalism, using the Fadeev-Popov procedure to fix both diffeomorphisms and the Weyl symmetry, the gauge fixing in Eq. (15) avoids mixed terms in the ghost system; the ghosts for the Weyl gauge fixing are non-dynamical and integrating them out is
}

\section{Conformal gravity}

We return to our physical issue: the coupling $f_{0}$ is not asymptotically free. In Sect. 4 we will argue that $f_{0}$ grows with energy, becoming non-perturbative at $f_{0} \sim 4 \pi$ and continuing to grow up to $f_{0} \rightarrow \infty$ in the limit of infinite energy, such that the $R^{2} / 6 f_{0}^{2}$ term disappears from the action. In this section we show that this limit is well defined. It is precisely defined as agravity with parameters chosen such that all Weyl-breaking terms $\mathscr{L}_{\text {WeyT }}$ in Eq. (5) vanish:

$f_{0}=\infty, \quad \xi_{a b}=-\frac{\delta_{a b}}{6}$.

The $R^{2} / 6 f_{0}^{2}$ term provides the kinetic term for $\sigma$, the conformal mode of the agraviton. Thereby $\sigma$ fluctuates wildly in the limit $f_{0} \rightarrow \infty$. Indeed, the agraviton propagator of [4] has a contribution proportional to $f_{0}^{2}$, which diverges as $f_{0} \rightarrow \infty$. Faddeev and Popov have shown how to deal with these situations: add an extra gauge fixing for the extra gauge redundancy appearing in conformal gravity, local Weyl transformations.

In general, conformal gravity is not a consistent quantum theory, because its Weyl gauge symmetry is anomalous. In a simpler language, the dimensionless couplings run with energy as described by their RGE. ${ }^{8}$ The theory is no longer scale invariant, and the conformal mode of the graviton couples to all non-vanishing $\beta$-functions. The Weyl-breaking terms of the agravity Lagrangian are generated back by quantum corrections. The consistent quantum theory is agravity. For this reason our work differs from articles where conformal gravity is proposed as a complete theory of gravity $[39,40]$.

Nevertheless, conformal gravity can be the consistent infinite-energy limit of agravity provided that all $\beta$-functions vanish at infinite energy: the theory must be asymptotically free or asymptotically safe, in other words all couplings other than $f_{0}$ have to reach a UV fixed point where all $\beta$-functions vanish, as we will see.

In this section we clarify these issues by computing the one-loop RGE of conformal gravity coupled to a generic matter sector, as in Eq. (4). The RGE can be obtained without performing any extra computation by using the perturbative equality obtained in the previous section: agravity is equivalent to conformal gravity plus two extra scalars, $\vec{\Omega}$. In the other direction, this means that conformal gravity has the same RGE as agravity minus two scalars. Thereby the

Footnote 7 continued

equivalent to the modified diffeomorphism transformation law of the traceless graviton, Eq. (22).

${ }^{8}$ One might hope that all couplings stay at fixed points at all energies, but this possibility is excluded because one must recover a nonconformal behavior at low energies for phenomenological reasons. 
RGE for $f_{2}$ in conformal gravity is obtained by substituting $N_{s} \rightarrow N_{s}-2$ in (50a) obtaining

$$
\begin{aligned}
(4 \pi)^{2} \frac{\mathrm{d} f_{2}^{2}}{\mathrm{~d} \ln \bar{\mu}}= & -f_{2}^{4}\left(\frac{199}{15}+\frac{N_{V}}{5}+\frac{N_{f}}{20}+\frac{N_{s}}{60}\right) \\
& \left(\text { for } f_{0} \rightarrow \infty \text { and } \xi_{a b} \rightarrow-\frac{1}{6} \delta_{a b}\right) .
\end{aligned}
$$

This reproduces the result obtained in [41-44] with a dedicated computation in the gauge of Eq. (13), where only the traceless part of the graviton propagates; see Eq. (16). Then the one-loop RGE for all other parameters can be obtained from the agravity RGE, listed in the appendix, by dropping those for $f_{0}$ and $\xi_{a b}$, as well as the terms involving $f_{0}$ and $\xi_{a b}+\delta_{a b} / 6$ from the remaining RGE. The result is

$$
\begin{aligned}
(4 \pi)^{2} \frac{\mathrm{d} Y^{a}}{\mathrm{~d} \ln \bar{\mu}}= & \frac{1}{2}\left(Y^{\dot{b}} Y^{b} Y^{a}+Y^{a} Y^{\dot{\dagger}} Y^{b}\right)+2 Y^{b} Y^{\dagger a} Y^{b} \\
& +Y^{b} \operatorname{Tr}\left(Y^{\dot{b}} Y^{a}\right)-3\left\{C_{2 F}, Y^{a}\right\}+\frac{15}{8} f_{2}^{2} Y^{a} \\
(4 \pi)^{2} \frac{\mathrm{d} \lambda_{a b c d}}{\mathrm{~d} \ln \bar{\mu}}= & \sum_{\text {perms }}\left[\frac{1}{8} \lambda_{a b e f} \lambda_{e f c d}\right. \\
& +\frac{3}{8}\left\{\theta^{A}, \theta^{B}\right\}_{a b}\left\{\theta^{A}, \theta^{B}\right\}_{c d}-\operatorname{Tr} Y^{a} Y^{\dot{\dagger} b} Y^{c} Y^{\dagger d} \\
& +\frac{5}{288} f_{2}^{4} \delta_{a b} \delta_{c d}+\lambda_{a b c d} \\
& \times\left[\sum_{k=a, b, c, d}\left(Y_{2}^{k}-3 C_{2 S}^{k}\right)+5 f_{2}^{2}\right]
\end{aligned}
$$

for $f_{0} \rightarrow \infty$ and $\xi_{a b} \rightarrow-\frac{1}{6} \delta_{a b}$, where $Y_{2}^{k}, C_{2 S}^{k}$ and $C_{2 F}$ are defined in Eq. (51). We do not know of any previous determinations of the RGE in (25) and (26). We do not show the RGE of the gauge couplings because they are not modified by the gravitational couplings (see the first paper in [41-44] and $[4,45,46])$.

\section{Anomalous generation of $1 / f_{0}^{2}$}

However, the fact that $f_{2}$ and other gauge, Yukawa and quartic couplings start having non-vanishing $\beta$-functions means that the conformal-gravity computation becomes inconsistent when going to higher orders. The conformal mode of the agraviton, $\sigma$, is a decoupled degree of freedom in the classical Lagrangian of conformal gravity. At quantum loop level, $\sigma$ starts coupling to all terms that break scale invariance proportionally to their $\beta$-functions, so that $\sigma$ can no longer be gauged away.

Once $\sigma$ couples to other particles, they can propagate in loops within Feynman diagrams containing, as external states, $\sigma$ only. This describes how the $R^{2}$ term is generated at a loop level sufficiently high for the diagram to contain running couplings. The result can be written in terms of $\beta$ functions through the aid of consistency conditions obtained by formally promoting the couplings to fields, including the gravitational coupling. A scalar quartic $\lambda$ starts contributing at $\lambda^{5}$ order [47,48]; a gauge interaction starts contributing at $g^{6}$ order [49,50]; the effect of scalar quartics, Yukawa and gauge couplings was computed in [51] in parity-invariant theories. The final result can be written as an RGE for $1 / f_{0}^{2}$ :

$$
\begin{aligned}
\frac{\mathrm{d}}{\mathrm{d} \ln \bar{\mu}} \frac{1}{f_{0}^{2}}= & \frac{b_{1} b_{2} N_{V}}{18} \frac{g^{6}}{(4 \pi)^{8}}+\frac{1}{25920(4 \pi)^{12}} \\
& \times\left(6 \lambda_{a b c d} \lambda_{c d m n} \lambda_{\text {mnpq }} \lambda_{\text {aprs }} \lambda_{\text {bqrs }}\right. \\
& +12 \lambda_{\text {abcd }} \lambda_{c d m n} \lambda_{\text {mrpq }} \lambda_{\text {bspq }} \lambda_{\text {anr }} \\
& \left.-\lambda_{\text {acdm }} \lambda_{\text {bcdm }} \lambda_{\text {anrs }} \lambda_{\text {bnpq }} \lambda_{\text {rsp }}\right)+\cdots
\end{aligned}
$$

in the limit $f_{0} \rightarrow \infty$ and $\xi_{a b} \rightarrow-\delta_{a b} / 6$. We have written explicitly the leading gauge contribution assuming, for simplicity, a gauge group $G$ with a single gauge coupling $g, N_{V}$ vectors and $N_{f}$ Weyl fermions in the same representation $R$ of $G: b_{1}$ and $b_{2}$ are the usual one-loop and two-loop $\beta$-function coefficients for $g$, precisely defined as $\mathrm{d} g / \mathrm{d} \ln \bar{\mu}=-b_{1} g^{3} /(4 \pi)^{2}-b_{2} g^{5} /(4 \pi)^{4}+\cdots$ and given by $[52]^{9}$ We also have

$$
\begin{aligned}
& b_{1}=\frac{11}{3} C_{2 G}-\frac{2}{3} T_{F} N_{f}, \\
& b_{2}=\frac{34}{3} C_{2 G}^{2}-\frac{10}{3} C_{2 G} T_{F} N_{f}-2 C_{2 F} T_{F} N_{f} .
\end{aligned}
$$

The gauge contribution to $1 / f_{0}^{2}$ can be either positive or negative, depending on the field content. For example, in the SM one has $N_{V}=3, b_{1}=19 / 6$ and $b_{2}=-35 / 6$ for $\mathrm{SU}(2)_{L}$ and $N_{V}=8, b_{1}=7$ and $b_{2}=26$ for $\mathrm{SU}(3)_{c}$. The quartic of the Higgs doubled $H$, defined by the potential $\lambda_{H}|H|^{4}$, contributes to the RGE for $1 / f_{0}^{2}$ as $416 \lambda_{H}^{5} / 5(4 \pi)^{12}$, which is sub-dominant with respect to the gauge contributions. Integrating the gauge contribution alone from infinite energy down to a scale where $g \ll 1$, one finds $1 / f_{0}^{2} \simeq-b_{2} N_{V} g^{4} / 72(4 \pi)^{6}$.

The ... in Eq. (27) denote extra terms due to Yukawa couplings (partially computed in [51]) and to gravitational terms (never computed and presumably first arising at order $f_{2}^{6}$ ). The full unknown expression might perhaps take the form of a $\beta$-function of some combination of couplings, given that the Weyl symmetry is not broken when all $\beta$-functions vanish. Barring this exception, which seems not to be relevant

9 The group quantities $C_{2 G}, C_{2 F}$ and $T_{F}$ are defined as usual in terms of the generators $t^{A}$ in the representation $R$ as follows:

$$
\begin{aligned}
{\left[t^{A}, t^{B}\right] } & =i f^{A B C} t^{C}, \quad f^{A C D} f^{B C D}=C_{2 G} \delta^{A B}, \\
t^{A} t^{A} & =C_{2 F}, \quad \operatorname{Tr}\left(t^{A} t^{B}\right)=T_{F} \delta^{A B} .
\end{aligned}
$$

For example, for the vector representation of $\mathrm{SU}(N)$ we have

$C_{2 G}=N, \quad C_{2 F}=\frac{N^{2}-1}{2 N}, \quad T_{F}=\frac{1}{2}$. 
(nature is neither described by a free theory nor by a conformal theory), Eq. (27) means that conformal gravity is not a complete theory: at some loop level, quantum corrections start generating back the extra couplings $f_{0}$ and $\xi_{a b}$ present in agravity.

One important aspect of Eq. (27) is that its right-hand side vanishes when all couplings sit at a fixed point, where all $\beta$-functions vanish. This tells us that the $f_{0} \rightarrow \infty$ limit is consistent when the other couplings on the right-hand-side approach a fixed point.

Anomalous generation of $\xi+1 / 6$

Non-conformal $\xi$-couplings are generated at one-loop by the gravitational coupling $f_{2}$. Starting from $\xi=-1 / 6$ at infinite energy, $f_{2}$ induces a negative value of

$f_{0}^{2}(\xi+1 / 6) \sim-\mathcal{O}\left(f_{2}^{2}\right)$

at finite energy. However, as argued later, naturalness demands $f_{2} \lesssim 10^{-8}$. At perturbative level, $f_{0}$ alone does not generate $\xi+1 / 6$. Extra anomalous contributions to $\xi+1 / 6$ are first generated at order $y^{6} /(4 \pi)^{6}, y^{2} \lambda^{2} /(4 \pi)^{6}, \lambda^{4} /(4 \pi)^{8}$ in the Yukawa couplings $y$ and in the scalar quartics $\lambda$ (see eqs. (6.33) and (7.22) of [51], where individual terms have different signs; see also $[47,48])$. For example the quartic couplings alone contribute as

$$
\begin{aligned}
\frac{\mathrm{d} \zeta_{a b}}{\mathrm{~d} \ln \bar{\mu}}= & \frac{1}{18(4 \pi)^{8}} \\
& \times\left(\frac{1}{6} \lambda_{c p q r} \lambda_{d p q r} \lambda_{c m n a} \lambda_{d m n b}+\lambda_{p q m n} \lambda_{p q c d} \lambda_{c m r a} \lambda_{d n r b}\right. \\
& \left.-\lambda_{r p q d} \lambda_{r m n c} \lambda_{d m n a} \lambda_{c p q b}\right)+\cdots
\end{aligned}
$$

for $f_{0} \rightarrow \infty$ and $\xi_{a b} \rightarrow-\delta_{a b} / 6$, where $\cdots$ denote the contribution of the other couplings. In the SM Higgs case this contribution is $\mathrm{d} \xi_{H} / \mathrm{d} \ln \bar{\mu}=48 \lambda_{H}^{4} /(4 \pi)^{8}+\cdots$, having written the potential as $\lambda_{H}|H|^{4}$ and the non-minimal coupling to gravity as $-\xi_{H}|H|^{2} R$.

It is important to note that the right-hand-side of Eq. (32) vanishes when all couplings sit at a fixed point, where all $\beta$-functions vanish. This tells us that the $f_{0} \rightarrow \infty$ limit is consistent when at the same time $\zeta_{a b} \rightarrow 0$ and the other couplings approach a fixed point. In this precise limit the conformal mode decouples from the rest of the degrees of freedom.

\section{The conformal mode of the agraviton}

So far we have shown that a large self-coupling $f_{0}$ of the conformal mode of the agraviton does not affect the rest of physics, provided that the non-minimal couplings $\xi$ of scalars go to the conformal value and the remaining couplings approach a fixed point. We next address the big issue: what happens to the conformal mode of the agraviton when $f_{0}$ is big?

The one-loop agravity RGE for $f_{0}$, Eq. (3), is valid for $f_{0} \ll 1$ and shows that a small $f_{0}$ grows with energy. In general, when a dimensionless coupling behaves in this way, three qualitatively different things can happen depending on the non-perturbative behavior of the $\beta$-function,

$$
\frac{\mathrm{d} f_{0}}{\mathrm{~d} \ln \bar{\mu}}=\beta\left(f_{0}\right) \text {. }
$$

1. If $\beta\left(f_{0}\right)$ grows at large $f_{0}$ faster than $f_{0}$, then $\int^{\infty} \mathrm{d} f_{0} /$ $\beta\left(f_{0}\right)$ is finite and $f_{0}$ hits a Landau pole at finite energy. The theory is inconsistent. ${ }^{10}$

2. If $\beta\left(f_{0}\right)$ vanishes for some $f_{0}=f_{0}^{*}$, then $f_{0}$ grows to $f_{0}^{*}$, entering into asymptotic safety.

3. If $\beta\left(f_{0}\right)$ remains positive but grows less than or as $f_{0}$, then $f_{0}$ grows to $f_{0}=\infty$ at infinite energy. ${ }^{11}$

In order to study what happens at large $f_{0}$, we can ignore all other couplings and focus on the conformal mode of the agraviton. We can choose a conformally flat background $g_{\mu \nu}(x)=e^{2 \sigma(x)} \eta_{\mu \nu}$, as the background does not affect the UV properties of the theory. Recalling Eq. (8), the action for the conformal mode only is

$$
\begin{aligned}
S & =\int \mathrm{d}^{4} x \sqrt{|\operatorname{det} g|} \frac{R^{2}}{6 f_{0}^{2}}=\frac{6}{f_{0}^{2}} \int \mathrm{d}^{4} x\left(e^{-\sigma} \square e^{\sigma}\right)^{2} \\
& =\frac{6}{f_{0}^{2}} \int \mathrm{d}^{4} x\left[\square \sigma+(\partial \sigma)^{2}\right]^{2} .
\end{aligned}
$$

The field $\sigma$ has mass dimension 0 , and its action in Eq. (34) respects the following symmetries: shifts $\sigma(x) \rightarrow \sigma(x)+$ $\delta \sigma$; Poincaré invariance; scale invariance; invariance under special conformal transformations:

$$
\begin{aligned}
\sigma(x) & \rightarrow \sigma\left(x^{\prime}\right)-2 c \cdot x, \\
x_{\mu}^{\prime} & =x_{\mu}+c_{\mu} x^{2}-2 x_{\mu}(c \cdot x),
\end{aligned}
$$

at first order in the infinitesimal constant vector $c_{\mu}$. Conformal invariance here appears as a residual of the reparametrization invariance of the gravitational theory: it is present because conformal transformations are those reparametrizations that leave the metric invariant, up to an overall scale factor. Being a residual of reparametrization invariance, this symmetry is non-anomalous, up to the usual scale anomaly. No other action is compatible with these symmetries. Taking

\footnotetext{
${ }^{10}$ For example, lattice simulations indicate that one scalar quartic or the gauge coupling in QED behave in this way [53-55].

${ }^{11}$ For example, this behavior is realized if the $\beta$-function has the form $\beta\left(f_{0}\right)=f_{0} Z\left(f_{0}\right)$ with $Z\left(f_{0}\right)=b /\left(f_{0}^{2}+f_{0}^{-2}\right) /(4 \pi)^{2}$ with $b>0$. Then at low energy $f_{0}$ runs logarithmically towards $f_{0} \rightarrow 0$, and at large energy $1 / f_{0}$ runs logarithmically towards $1 / f_{0} \rightarrow 0$. Indeed, the full solution for $f_{0}^{2}>0$ is $f_{0}^{2}=t+\sqrt{1+t^{2}}$ where $t=b \ln \left(\bar{\mu} / \Lambda_{0}\right) /(4 \pi)^{2}$ and $\Lambda_{0}$ is the transition scale at which $f_{0} \sim 1$.
} 
into account that $\mathrm{d}^{4} x=(1+8 c \cdot x) \mathrm{d}^{4} x^{\prime}$, the single terms in the action of Eq. (34) vary under a conformal transformation as

$\delta \int \mathrm{d}^{4} x(\partial \sigma)^{4}=8 \int \mathrm{d}^{4} x\left[-c \cdot \partial \sigma(\partial \sigma)^{2}\right]$,

$\delta \int \mathrm{d}^{4} x(\partial \sigma)^{2} \square \sigma=4 \int d^{4} x\left[c \cdot \partial \sigma(\partial \sigma)^{2}-c \cdot \partial \sigma \square \sigma\right]$,

$\delta \int \mathrm{d}^{4} x(\square \sigma)^{2}=8 \int \mathrm{d}^{4} x[c \cdot \partial \sigma \square \sigma]$,

such that the combination in Eq. (34) is invariant. ${ }^{12}$ We verified, at tree level, that the scattering amplitudes vanish, in agreement with the Coleman-Mandula theorem.

For small $f_{0}$ one can compute the theory perturbatively around the four-derivative kinetic term $(\square \sigma)^{2}$. As discussed in Sect. 2, this can be equivalently formulated as an $\mathrm{SO}(2)$ invariant scalar $\Omega$ with a quartic coupling. This shows that UV-divergent quantum corrections preserve the form of the action, such that the quantum action is given by

$\Gamma=Z\left(f_{0}\right) S+$ finite effects.

Indeed, in the scalar theory with the field $\Omega$ and the simple quartic coupling all divergences can be reabsorbed by renormalizing $f_{0}^{2}$ (which in that formulation represents the quartic coupling) and the field, $\Omega$. Going back to the formulation in terms of $\sigma$, both renormalizations (of $f_{0}$ and of $\Omega$ ) can be expressed in terms of a common rescaling of the action, which is what appears in Eq. (37).

The common UV-divergent factor $Z\left(f_{0}\right)$ renormalizes equally all terms in the action, such that it can be seen as an RGE running of $f_{0}$, which we give here up to two loops:

$\frac{\mathrm{d} f_{0}^{2}}{\mathrm{~d} \ln \bar{\mu}}=\frac{1}{(4 \pi)^{2}} \frac{5}{6} f_{0}^{4}-\frac{1}{(4 \pi)^{4}} \frac{5}{12} f_{0}^{6}+\cdots$.

The one-loop term reproduces the corresponding term in the full gravitational computation, Eq. (3), while the twoloop term was never obtained before. The Weyl anomaly, mentioned in Sect. 2, affects this RGE only at higher loop level. The reason is that the $\beta$-functions are already one-loop effects, so that one needs at least two vertices and one extra loop to get a contribution from the anomaly. This remark not only applies to pure anomalous effects, but also to mixed $f_{0}$-anomaly contributions; in the latter case, indeed, a couple of internal $\sigma$-lines should be converted to the particles

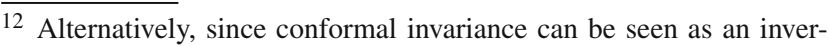
sion $x^{\mu} \rightarrow y^{\mu}=x^{\mu} / x^{2}$ followed by a translation and by another inversion, one can more simply check that the action is invariant under the inversion: $\mathrm{d}^{4} x \rightarrow \mathrm{d}^{4} y / y^{8}, \sigma(x) \rightarrow \sigma(y)+\ln y^{2}$ and $\left[\square_{x} \sigma+\left(\partial_{x} \sigma\right)^{2}\right]=y^{4}\left[\square_{y} \sigma+\left(\partial_{y} \sigma\right)^{2}\right]$. The transformation rule of $\sigma$ under the coordinate transformation $x^{\mu} \rightarrow y^{\mu}=x^{\mu} / x^{2}$ can be obtained by recalling its general definition in (6) and that we are assuming here a conformally flat metric, i.e. $g_{\mu \nu}(x)=e^{2 \sigma(x)} \eta_{\mu \nu}$.
}

which $\sigma$ couples to through the anomaly and again at least two vertices proportional to $\beta$-functions and one extra loop are needed.

When $f_{0}$ grows the path integral receives contributions from fluctuations of $\sigma$ with larger and larger amplitude, probing the terms in the action of Eq. (34) with higher powers in $\sigma$. For large $f_{0}$ the action becomes dominated by the $(\partial \sigma)^{4}$ term that has the highest power of $\sigma$, while the kinetic term becomes negligible. This can happen because all terms in the action have the same number of derivatives. For example, a field configuration $\sigma(r)=\sigma_{0} e^{-r^{2} / a^{2}}$ contributes as $S \sim\left(\sigma_{0}+\sigma_{0}^{2}\right)^{2} / f_{0}^{2}$, independently of the scale $a$, such that for $f_{0} \gtrsim 1$ the path integral is dominated by the second term.

In the limit $f_{0} \rightarrow \infty$ the action $S$ simplifies to

$S_{\infty}=\frac{6}{f_{0}^{2}} \int \mathrm{d}^{4} x(\partial \sigma)^{4}$.

Although for large $f_{0}$ the theory is non-perturbative in $f_{0}$, one can still develop an analytical argument to show the absence of a Landau pole of $f_{0}$, as we now discuss. The action in Eq. (39) acquires new symmetries: $S_{\infty}$ is $\mathbb{Z}_{2}$-invariant $\left(\mathbb{Z}_{4}\right.$ invariant if complexified); furthermore, being the term of $S$ with the highest power of $\sigma$, it is invariant under the homogeneous part of the transformation in Eq. (35), while the other two terms, $(\partial \sigma)^{2} \square \sigma$ and $(\square \sigma)^{2}$ or any combination of them, are not. Symmetries imply that the quantum action $\Gamma_{\infty}$, which includes the classical and UV-divergent quantum corrections, is fully described by $\Gamma_{\infty}=Z_{\infty} S_{\infty}$, where $Z_{\infty}$ is a constant, related to the $Z\left(f_{0}\right)$ in the full theory as $Z_{\infty}=\lim _{f_{0} \rightarrow \infty} Z\left(f_{0}\right)$. This constant must equal unity, $Z_{\infty}=1$ because the theory is classical at large field values, for which $S_{\infty} \gg 1$, and because its form at all field values is fixed by symmetries. The theory with action $S_{\infty}$, despite being interacting, behaves as a free theory, in the sense that the quantum action does not receive divergent corrections.

This shows that, in the full theory, $f_{0}$ can flow to large values without hitting Landau poles: $\beta\left(f_{0}\right)=\mathcal{O}\left(1 / f_{0}\right)$ at $f_{0} \gg 1$. Having distilled the non-perturbative dynamics of the conformal mode of the agraviton in a simple action, Eq. (34), it seems now feasible to fully clarify its dynamics. We have shown that it hits no Landau poles, excluding case 1 . of the initial list. The theory at $f_{0} \gg 1$ should be computable by developing a perturbation theory in $1 / f_{0}$. We have not been able of excluding case 2 : a vanishing $\beta\left(f_{0}\right)$ at $f_{0} \sim 4 \pi$. Nonperturbative numerical techniques seem needed to determine the behavior of the theory at the intermediate energy at which $f_{0} \sim 4 \pi$, although this currently needs adding a regulator that breaks the symmetries of the theory (such as a lattice or a momentum averager [56-58]), obscuring possible general properties (such as the sign of $\beta\left(f_{0}\right)$ ) that could follow from the positivity of the symmetric action in Eq. (34). 
The letter ' $a$ ' in the name 'conformal mode of the agraviton' reminds us that our field $\sigma$ contains two degrees of freedom because its action contains four derivatives, while the usual 'conformal mode of the graviton' obtained from the Einstein action only contains one degree of freedom. More precisely, the Einstein term alone, $-\frac{1}{2} \bar{M}_{\mathrm{Pl}}^{2} R$, where $\bar{M}_{\mathrm{Pl}}$ is the reduced Planck mass, gives a negative kinetic term $3 \bar{M}_{\mathrm{Pl}}^{2} \Omega_{L} \square \Omega_{L}$ for $\Omega_{L}=e^{\sigma}$; see Eq. (7). Summing the Einstein term with $R^{2} / 6 f_{0}^{2}$, the four-derivative conformal mode of the agraviton $\sigma$ splits into a physical mode with positive kinetic term and mass $M_{0}=f_{0} \bar{M}_{\mathrm{Pl}} / \sqrt{2}$ for $f_{0} \ll 1$, and the usual massless Einstein term, which is reparametrization dependent. ${ }^{13}$ To see this, it is convenient to use the form of the action where $\sigma$ is rewritten in terms of two fields with two derivatives, $\Omega_{L}$ and $\Omega_{R}$ (see Sect. 2). Adding to the previous discussion the Planck mass the Lagrangian becomes

$\mathscr{L}=-2 \Omega_{R} \square \Omega_{L}-\frac{1}{6} f_{0}^{2} \Omega_{L}^{2} \Omega_{R}^{2}+3 \bar{M}_{\mathrm{Pl}}^{2} \Omega_{L} \square \Omega_{L}$.

We expand in fluctuations around the minimum, $\Omega_{R}=0$ and $\Omega_{L}=1$, where we arbitrarily choose unity in order to keep the metric as $\eta_{\mu \nu}$, while other values would correspond to a different unit of mass. Then the quadratic part of the action can be diagonalized by defining $\Omega_{L}=1+(\alpha+\beta) / \sqrt{3} \bar{M}_{\mathrm{Pl}}$, $\Omega_{R}=\sqrt{3} M \beta$, where $\alpha$ is the Einstein ghost and $\beta$ is the massive scalar component of the graviton. The result is

$$
\begin{aligned}
& \mathscr{L}=\alpha \square \alpha+\beta\left(-\square-M_{0}^{2}\right) \beta-V \quad \text { with } \\
& V=\frac{1}{6} f_{0}^{2} \beta^{2}(\alpha+\beta)\left(\alpha+\beta+2 \sqrt{3} \bar{M}_{\mathrm{Pl}}\right) .
\end{aligned}
$$

\section{Scenarios compatible with naturalness of the Higgs mass}

In the following we discuss implications of case 3. Qualitatively different scenarios can arise, depending on the ordering between the key scales:

- $\Lambda_{0}$, the energy scale at which the self-coupling of the conformal mode equals $f_{0} \sim 4 \pi$, with $f_{0} \ll 4 \pi$ at $E \ll \Lambda_{0}$ and $f_{0} \gg 4 \pi$ at $E \gg \Lambda_{0}$.

- $\Lambda_{2}$, the energy scale at which the graviton self-coupling equals $f_{2} \sim 4 \pi$, with $f_{2} \ll 4 \pi$ at $E \gg \Lambda_{2}$.

- The Planck scale. As this is the largest known mass scale, in the context of dimensionless theories it can be

\footnotetext{
${ }^{13}$ Many authors refuse to view the theory with higher derivative as legitimate because of the consequent ghosts; see e.g. [59] for attempts to discard the $(\square \sigma)^{2}$ term. Accepting the presence of higher derivatives allows one to describe the Weyl anomaly as ordinary RGE running of $f_{0,2}$, rather than by modifying Einstein gravity by adding a complicated 'quantum anomalous action' [60-62] which encodes the anomalous behavior of generic undefined theories of gravity.
}

interpreted as the largest dynamically generated vacuum expectation value or condensate.

The scales $\Lambda_{0,2}$ can be physically realized in nature (like the scale $\Lambda_{\mathrm{QCD}}$ at which $\mathrm{SU}(3)_{c}$ becomes strong) if they are larger than the Planck scale. Otherwise they are not realized (like the scale at which $\mathrm{SU}(2)_{L}$ would have become strong, if symmetry breaking had not occurred at a higher energy) and we use $\Lambda_{2} \ll M_{\mathrm{Pl}}$ to denote $f_{2} \ll 1$ at $M_{\mathrm{Pl}}$ where $M_{\mathrm{Pl}}$ is the Planck mass.

In this section we adopt Higgs mass naturalness as a criterion to limit the possible speculations. For example, the simplest possibility in which the Planck scale is identified with $\Lambda_{2}$ or $\Lambda_{0}$ leads to unnaturally large physical corrections to the Higgs mass from gravity. Naturalness demands $f_{2} \ll 1$ at the Planck scale, while $f_{0}$ can be either very small or very large, giving rise to two natural possibilities shown in Fig. 1: $f_{0} \ll 1$ at $M_{\mathrm{Pl}}$ (left panel) and $f_{0} \gg 1$ at $M_{\mathrm{Pl}}$ (right).

\section{$5.1 f_{0} \ll 1$ at the Planck scale}

The first possibility is the one considered in [4], which showed that the Planck mass can be dynamically generated, within a dimensionless theory, from a dynamically induced vacuum expectation value of a fundamental scalar $S=\left(s+i s^{\prime}\right) / \sqrt{2}$. The part of the dimensionless Lagrangian involving $S$ and the SM Higgs doublet $H$ is

$$
\begin{aligned}
\mathscr{L}= & {\left[\left|D_{\mu} S\right|^{2}-\lambda_{S}|S|^{4}-\xi_{S}|S|^{2} R\right] } \\
& +\left[\left|D_{\mu} H\right|^{2}-\lambda_{H}|H|^{4}-\xi_{H}|H|^{2} R\right] \\
& +\lambda_{H S}|S|^{2}|H|^{2} .
\end{aligned}
$$

Provided that $\lambda_{S}$ runs in such a way that it vanishes at the same scale at which its $\beta$-function vanishes, $s$ gets a vacuum expectation value with cosmological constant tuned to zero, and $\bar{M}_{\mathrm{Pl}}^{2}=\xi_{S}\langle s\rangle^{2}$ is positive provided that the parameter $\xi_{S}$, renormalized at the Planck scale, is positive. An unpleasant feature of the model is that the mixed quartic $\lambda_{H S}$ must be very small, in order to avoid inducing an unnaturally large contribution to the Higgs mass $\left(M_{h}^{2}=\lambda_{H S}\langle s\rangle^{2}\right.$, which appears in the potential as $\left.-M_{h}^{2}|H|^{2} / 2\right)$. References $[4,6,7]$ showed that $\lambda_{H S}$ can be naturally small, despite being generated at loop level through gravity loops as

$$
\begin{aligned}
(4 \pi)^{2} \frac{\mathrm{d} \lambda_{H S}}{\mathrm{~d} \ln \bar{\mu}}= & -\xi_{H} \xi_{S}\left[5 f_{2}^{4}+36 \tilde{\lambda}_{H} \tilde{\lambda}_{S}\right] \\
& +\cdots \quad\left(f_{0} \ll 1\right),
\end{aligned}
$$

where $\tilde{\lambda}_{S} \equiv f_{0}^{2}\left(\xi_{S}+1 / 6\right)$ and $\tilde{\lambda}_{H} \equiv f_{0}^{2}\left(\xi_{H}+1 / 6\right)$ are the couplings that appear in the perturbatively equivalent formulation of agravity of Eq. (10), where $f_{0}$ and $\xi_{H, S}$ become quartic couplings with an extra scalar $\vec{\Omega}$. The Higgs mass 


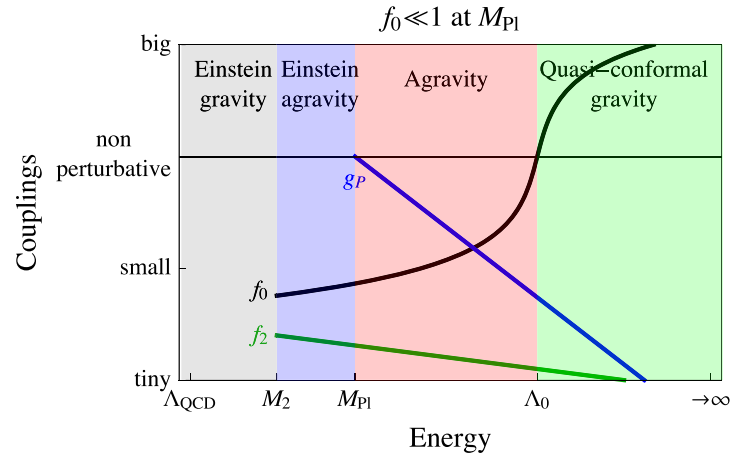

Fig. 1 RGE running of the main dimensionless couplings $f_{0}, f_{2}, g_{P}$ in the two possible scenarios that do not lead to unnaturally large corrections to the Higgs mass: $f_{0}, f_{2} \ll 1$ at the Planck scale (left), $f_{2} \ll 1$

is natural if $f_{0,2} \lesssim 10^{-8}$. The above scenario needs to be reconsidered:

(a) Is naturalness still satisfied, or $f_{0}$ becoming strongly coupled at the energy scale $\Lambda_{0}$ generates a $\tilde{\lambda}_{H, S}$ of the same order?

(b) Can one get $\xi_{S}>0$ at the Planck scale starting from $\xi_{S}=-1 / 6$ at infinite energy?

A peculiar RG running behavior at a very large scale, such as $\Lambda_{0} \gtrsim 10^{10^{16}} \mathrm{GeV}$, does not imply perturbative contributions to scalar masses of the same order, as long as no new physics nor vacuum expectation values nor condensates develop at that scale [25]. Non-perturbative ultra-Planckian contributions to the cosmological constant and the Planck mass from a $f_{0} \sim 4 \pi$ are forbidden by the global shift symmetry $\sigma \rightarrow \sigma+\delta \sigma$. Planckian corrections to the cosmological constant remain unnaturally large as usual.

The answer to (a) seems to be positive: as shown in Sect. 2 perturbative corrections in $f_{0}$ behave like quartic scalar couplings, and thereby renormalize the $\tilde{\lambda}_{H, S}$ couplings (mixed quartics between the scalars and the conformal mode of the graviton) only multiplicatively, like in the one-loop RGE, Eq. (50d). The same happens at $f_{0} \gg 1$ : non-vanishing $\tilde{\lambda}_{H, S}$ are only generated by $f_{2}$ (see Eq. (31)) and by the multi-loop anomalous effects discussed in Sect. 3. Non-perturbative corrections in $f_{0} \sim 4 \pi$ presumably too renormalize $\tilde{\lambda}_{H, S}$ only multiplicatively, as the scalars $H, S$ are not involved in the strong self-coupling of the conformal mode of the graviton.

Concerning issue (b), the answer can be positive in a theory where $\xi_{S}$ is very close to $-1 / 6$ around and above the energy scale $\Lambda_{0}$, and a positive $\xi_{S}$ is only generated through anomalous running (see e.g. Eq. (32)) at a much lower energy where $f_{0} \ll 1$ by some matter coupling becoming nonperturbative.

Given that non-perturbative physics seems anyhow necessary, we propose here a simpler mechanism for the generation

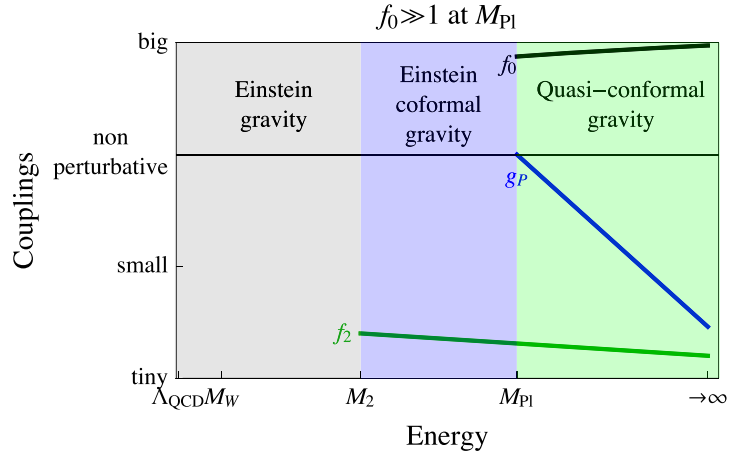

and $f_{0} \gg 1$ at the Planck scale (right). Here $M_{\mathrm{Pl}}$ is the Planck mass, $M_{2} \equiv f_{2} \bar{M}_{\mathrm{Pl}} / \sqrt{2}$ is the graviton ghost mass, $\Lambda_{0}$ is the RGE scales at which $f_{0} \sim 4 \pi$.

of the Planck mass that relies on a new strong coupling $g_{P}$, rather than on a perturbative coupling $\lambda_{S}$. Without introducing any extra scalar $S$ (and thereby bypassing the issue of a small $\lambda_{H S}$ ), the Planck scale can be induced by a new gauge group $G$ (under which the Higgs is neutral) with a gauge coupling $g_{P}$ that runs to non-perturbative values around the Planck scale, such that condensates $f$ are generated. This is shown as blue curve in Fig. 1. This scenario can be very predictive, as one coupling $g_{P}$ dominates the dynamics. The sign of $M_{\mathrm{Pl}}^{2}$ is predicted; however, it is not determined by dispersion relations and seems to depend on the detailed strong dynamics of the model (gauge group, extra matter representations) [63-68].

One has the desired $M_{\mathrm{Pl}}^{2}>0$ provided that the theory admits an effective-theory approximation where the effect of the strong dynamics is dominantly encoded in a mixing of the graviton with a composite spin-2 resonance, analogously to how a photon $/ \rho$ mixing approximates QCD effects. Then the relevant effective Lagrangian for the graviton $h_{\mu \nu}$ and the spin-2 resonance is

$$
\begin{aligned}
\mathscr{L}_{\text {eff }}= & -\frac{M^{2}}{2} R_{\rho}+f^{4}\left[a\left(h_{\mu \nu}-\rho_{\mu \nu}\right)^{2}\right. \\
& \left.+\left(h_{\mu}^{\mu}-\rho_{\mu}^{\mu}\right)^{2}\right]+\mathcal{O}\left(\partial^{4} h_{\mu \nu}\right)+\mathcal{O}\left(\partial^{4} \rho_{\mu \nu}\right) .
\end{aligned}
$$

The first term is the positive quadratic kinetic energy for the spin-2 resonance generated by strong dynamics; we wrote it as a 'curvature' $R_{\rho}$ multiplied by some positive $M^{2}>0$. The second term is a mass term, which presumably approximatively has Fierz-Pauli form, $a \approx 1 .{ }^{14}$ Next, we integrate out $\rho_{\mu \nu}$ obtaining an effective action for the graviton $h_{\mu \nu}$. At leading order in derivatives one simply has $\rho_{\mu \nu}=h_{\mu \nu}$, irrespectively of the precise form of the mass term. Thereby

\footnotetext{
$\overline{14}$ It can be rewritten in a covariant form as the mass term resulting, in the unitary gauge, from the spontaneous symmetry breaking of general coordinate invariance acting separately on ordinary fields and on composite fields, $\mathrm{GL}_{h} \otimes \mathrm{GL}_{\rho} \stackrel{f}{\rightarrow} \mathrm{GL}$ (see e.g. [69]).
} 
the resulting effective action is the Einstein action, with $\bar{M}_{\mathrm{Pl}}^{2}=M^{2}$.

Furthermore, the strong dynamics generates at the same time a cosmological constant. In a theory with no matter charged under $G$ it is negative and of order $M_{\mathrm{Pl}}^{4}$ :

$V=\frac{T_{\mu}^{\mu}}{4}=\frac{\partial_{\mu} \mathscr{D}^{\mu}}{4}=\frac{1}{4} \frac{\beta_{g_{P}}}{2 g_{P}}\left\langle F_{\alpha \beta}^{A 2}\right\rangle$

where $\mathscr{D}_{\mu}$ is the anomalous dilatation current and $\beta_{g_{P}}<0$.

This large contribution to the cosmological constant can be avoided if the theory also includes a Weyl fermion $\lambda$ in the adjoint of the gauge group $G$, such that the most general dimensionless action,

$$
\begin{aligned}
S= & \int \mathrm{d}^{4} x \sqrt{|\operatorname{det} g|} \\
& {\left[-\frac{F_{\mu \nu}^{A 2}}{4 g_{P}^{2}}+\bar{\lambda}^{A} i \not D \lambda^{A}+\frac{R^{2}}{6 f_{0}^{2}}+\frac{\frac{1}{3} R^{2}-R_{\mu \nu}^{2}}{f_{2}^{2}}\right], }
\end{aligned}
$$

is accidentally supersymmetric in its strongly coupled sector. With this particle content $\left\langle F_{\alpha \beta}^{A 2}\right\rangle=0$ vanishes, being the $D$ term of an accidental unbroken global supersymmetry, while the fermion condensate can be computed [70-72].

The Higgs has no renormalizable interaction with the strong sector at the Planck scale: it is only generated through gravitational loops, between the Planck mass and the masses $M_{0,2}$ of the extra components of the agraviton. The one-loop RGE for the Higgs mass in this regime was computed in [4], and the contribution proportional to $\bar{M}_{\mathrm{Pl}}^{2}$ is

$$
\begin{aligned}
(4 \pi)^{2} \frac{\mathrm{d}}{\mathrm{d} \ln \bar{\mu}} M_{h}^{2}= & -\xi_{H}\left[5 f_{2}^{4}+f_{0}^{4}\left(1+6 \xi_{H}\right)\right] \\
& \bar{M}_{\mathrm{Pl}}^{2}+\cdots \text { for } \quad M_{0,2}<\bar{\mu}<M_{\mathrm{Pl}}
\end{aligned}
$$

where $\cdots$ are contributions that are not dangerous from the point of view of naturalness. In Appendix B we write the one-loop RGE for the most general massive parameters.

\section{$5.2 f_{0} \gg 1$ at the Planck scale}

A simpler alternative that avoids having a very large RGE scale at which $f_{0}$ crosses $4 \pi$ is that $f_{0}$ is still large at the Planck scale and never gets small.

The conformal mode of the agraviton only has small anomalous couplings, until its dynamics suddenly changes when some vacuum expectation value or condensate is first generated. We assume that the largest such effect is the Planck mass, which can be generated in the ways discussed in the previous section. Then the tree-level Lagrangian of Eq. (41) describes how $\sigma$ splits into two-derivative modes. The $\mathrm{SO}(1,1)$ symmetry that prevented quantum corrections to the strongly interacting theory with $f_{0} \gg 1$ gets broken by $M_{\mathrm{Pl}}$.
The physical difference with respect to the previous case is that only the Einstein conformal mode of the graviton appears in the effective theory below the Planck scale down to the scale $M_{2}$. The RGE are those of gauge-fixed conformal gravity (see Eqs. (24), (25) and (26)). Proceeding as in Appendix B, the RGE of the Higgs mass is

$$
\begin{aligned}
(4 \pi)^{2} \frac{\mathrm{d}}{\mathrm{d} \ln \bar{\mu}} M_{h}^{2}= & \frac{5}{6} f_{2}^{4} \bar{M}_{\mathrm{Pl}}^{2}+\cdots, \\
& \text { for } \quad M_{2}<\bar{\mu}<M_{\mathrm{Pl}},
\end{aligned}
$$

which is naturally small for $f_{2} \lesssim 10^{-8}$.

\section{Conclusions}

In dimensionless gravity theories (agravity), the conformal mode of the agraviton consists of two fields: the usual conformal mode of the graviton and an extra scalar, jointly described by a four-derivative action for a single field $\sigma$, defined by $g_{\mu \nu}(x)=e^{2 \sigma(x)} \eta_{\mu \nu}$. The self-interactions of the conformal mode of the agraviton are controlled by a coupling $f_{0}$ that is not asymptotically free. In Sect. 2 we recomputed its RGE, and we extended it at the two-loop level, by developing a formulation where $f_{0}^{2}$ becomes an extra scalar quartic coupling. In the presence of scalars, their dimensionless $\xi$-couplings to gravity become scalar quartics, and the whole agravity can be rewritten as conformal gravity plus two extra scalars with an $\mathrm{SO}(1,1)$ symmetry. This perturbative equivalence allowed us to recompute the one-loop RGE equations of a generic agravity theory, confirming previous results [4], writing them in an equivalent simpler form where no couplings appear at the denominator in the $\beta$-functions, extending them at two loops.

In particular, rewriting $f_{0}^{2}$ as a quartic scalar clarifies why a small $f_{0}$ grows with energy in any agravity theory. A Landau pole would imply that agravity is only an effective theory and that the Higgs mass receives unnaturally large corrections.

In Sects. 2, 3 and 4 we have shown that, nevertheless, agravity can be a complete theory. Agravity can be extrapolated up to infinite energy, although in an unusual way: the dimensionless coupling $f_{0}$ grows with energy, becomes strongly coupled above some critical RGE scale $\Lambda_{0}$, and can smoothly grow to $f_{0} \rightarrow \infty$ at infinite energy. Although we have excluded that $f_{0}$ has a Landau pole, i.e. that it blows up at finite energy, there is another possibility which we have not studied in the present work: $f_{0}$ can approach asymptotically a finite non-perturbative fixed point. Analyzing this possibility requires having control on intermediate regimes where $f_{0} \sim 4 \pi$, which is beyond our current ability.

Provided that all scalars are asymptotically conformally coupled (all $\xi$-couplings must run approaching $-1 / 6$ ) and all matter couplings approach a fixed point (possibly a free one, like in QCD) in the UV, the simultaneous $f_{0} \rightarrow \infty$ limit 
turned out to be consistent. In this case and in the limit of infinite energy the conformal mode of the agraviton fluctuates freely and decouples from the rest of the theory. In the UV limit the theory can then be computed by viewing $\sigma$ as a gauge redundancy, which can be fixed with the FaddeevPopov procedure. One then obtains conformal gravity at infinite energy. In Sect. 3 we provided the one-loop RGE at the zero order in the expansion in $1 / f_{0}^{2}$ and $\xi+1 / 6$, including the most general matter sector.

However, the conformal symmetry is anomalous and its violation is dictated by renormalization group equations that describe how the dimensionless parameters that break conformal symmetry, $f_{0}$ and $\xi+1 / 6$, are generated at a few-loop order. As a result, at energies much above $\Lambda_{0}$ the conformal mode of the agraviton $\sigma$ is strongly self-coupled $\left(f_{0} \gg 1\right)$ and fluctuates wildly, being negligibly coupled to other particles. In Sect. 4 we isolated its peculiar action and showed that, despite the strong coupling, it can be controlled through its symmetries. The action is sufficiently simple for its full quantum behavior to be simulated on a Euclidean lattice.

The anomalous multi-loop RGE which generate $1 / f_{0}^{2}$ and $\xi+1 / 6$, are not (yet) fully known, but it is already possible to discuss the physical implications of this theory. We assume that the largest mass scale dynamically generated through vacuum expectation values or condensates is the Planck scale. Two situations discussed in Sect. 5 can lead to a scenario where the Higgs mass does not receive unnaturally large corrections. If $f_{0} \ll 1$ at the Planck scale one obtains agravity at sub-Planckian energies: we wrote the most general RGE for massive parameters, and we argued that a new gauge group with a fermion in the adjoint can become strongly coupled around the Planck scale and successfully generate $\bar{M}_{\mathrm{Pl}}$, without generating a Planckian cosmological constant (this mechanism was never explored before in the context of agravity). Alternatively, $f_{0} \gg 1$ at the Planck scale seems to be a viable possibility: in this case the scalar component of the agraviton is above the Planck scale.

Acknowledgements We thank Andrei O. Barvinsky, Agostino Patella, Alberto Ramos, Francesco Sannino, Ilya L. Shapiro, Andreas Stergiou, Nikolaos Tetradis, Enrico Trincherini and Hardi Veermae for useful discussions. This work was supported by the ERC grant NEO-NAT.

Open Access This article is distributed under the terms of the Creative Commons Attribution 4.0 International License (http://creativecomm ons.org/licenses/by/4.0/), which permits unrestricted use, distribution, and reproduction in any medium, provided you give appropriate credit to the original author(s) and the source, provide a link to the Creative Commons license, and indicate if changes were made. Funded by $\mathrm{SCOAP}^{3}$.

\section{A One-loop RGE in agravity}

When $f_{0}$ and all couplings are small, the one-loop $\beta$ functions $\beta_{p} \equiv d p / d \ln \bar{\mu}$ of all parameters $p$ of the generic agravity theory of Eqs. (4) and (5), can be conveniently written in terms of the combination of parameters that appear in Eq. (10), $\zeta_{a b}=\xi_{a b}+\delta_{a b} / 6$ and

$$
\begin{aligned}
\tilde{\lambda}_{a b c d} & =\lambda_{a b c d}+3 f_{0}^{2}\left(\zeta_{a b} \zeta_{c d}+\zeta_{a c} \zeta_{b d}+\zeta_{a d} \zeta_{b c}\right) \\
\tilde{\lambda}_{a b} & =f_{0}^{2} \zeta_{a b}, \quad \tilde{\lambda}=f_{0}^{2} .
\end{aligned}
$$

The RGE are

$$
(4 \pi)^{2} \frac{\mathrm{d} f_{2}^{2}}{\mathrm{~d} \ln \bar{\mu}}=-f_{2}^{4}\left(\frac{133}{10}+\frac{N_{V}}{5}+\frac{N_{f}}{20}+\frac{N_{s}}{60}\right)
$$

where $N_{V}, N_{f}, N_{s}$ are the number of vectors, Weyl fermions and real scalars (in the SM $N_{V}=12, N_{f}=45, N_{S}=4$ ), and

$$
\begin{aligned}
(4 \pi)^{2} \frac{\mathrm{d} f_{0}^{2}}{\mathrm{~d} \ln \bar{\mu}}= & \frac{5}{3} f_{2}^{4}+5 f_{2}^{2} f_{0}^{2}+\frac{5}{6} f_{0}^{4}+3 \tilde{\lambda}_{a b} \tilde{\lambda}_{a b}, \\
(4 \pi)^{2} \frac{\mathrm{d} \tilde{\lambda}_{a b c d}}{\mathrm{~d} \ln \bar{\mu}}= & \sum_{\text {perms }}\left[\frac{1}{8} \tilde{\lambda}_{a b e f} \tilde{\lambda}_{e f c d}\right. \\
& +\frac{3}{8}\left\{\theta^{A}, \theta^{B}\right\}_{a b}\left\{\theta^{A}, \theta^{B}\right\}_{c d} \\
& -\operatorname{Tr} Y^{a} Y^{\dagger b} Y^{c} Y^{\dagger d}+ \\
& \left.+\frac{5}{288} f_{2}^{4} \delta_{a b} \delta_{c d}+\frac{1}{16} \tilde{\lambda}_{a b} \tilde{\lambda}_{c d}\right] \\
& +\tilde{\lambda}_{a b c d}\left[5 f_{2}^{2}+\sum_{k=a, b, c, d}\left(Y_{2}^{k}-3 C_{2 S}^{k}\right)\right]
\end{aligned}
$$

$$
\begin{aligned}
(4 \pi)^{2} \frac{\mathrm{d} \tilde{\lambda}_{a b}}{\mathrm{~d} \ln \bar{\mu}}= & \frac{f_{0}^{2} \tilde{\lambda}_{a b}}{3}+\tilde{\lambda}_{a b c d} \tilde{\lambda}_{c d}+5 f_{2}^{2} \tilde{\lambda}_{a b} \\
& +\tilde{\lambda}_{a b} \sum_{k=a, b}\left(Y_{2}^{k}-3 C_{2 S}^{k}\right)+2 \tilde{\lambda}_{a c} \tilde{\lambda}_{c b} \\
& +\frac{5 f_{2}^{4} \delta_{a b}}{18}, \\
(4 \pi)^{2} \frac{d Y^{a}}{d \ln \bar{\mu}}= & \frac{Y^{\dagger b} Y^{b} Y^{a}+Y^{a} Y^{\dagger b} Y^{b}}{2} \\
& +2 Y^{b} Y^{\dagger a} Y^{b}+Y^{b} \operatorname{Tr}\left(Y^{\dagger b} Y^{a}\right) \\
& -3\left\{C_{2 F}, Y^{a}\right\}+\frac{15}{8} f_{2}^{2} Y^{a}
\end{aligned}
$$

The sum over "perms" runs over the 4 ! permutations of $a b c d$ and $Y_{2}^{k}, C_{2 S}^{k}$ and $C_{2 F}$ are defined by

$$
\begin{aligned}
\operatorname{Tr}\left(Y^{\dagger a} Y^{b}\right) & =Y_{2}^{a} \delta^{a b}, \quad \theta_{a c}^{A} \theta_{c b}^{A}=C_{2 S}^{a} \delta_{a b}, \\
C_{2 F} & =t^{A} t^{A}
\end{aligned}
$$

where $\theta^{A}$ and $t^{A}$ are the generators of the gauge group for scalars and fermions, respectively (the gauge couplings are contained in $\theta^{A}$ and $t^{A}$ ). 


\section{B One-loop RGE for massive parameters in agravity}

For the sake of completeness we also write the RGE for the most generic massive parameters that can be added while keeping the theory renormalizable: the reduced Planck mass $\bar{M}_{\mathrm{Pl}}=M_{\mathrm{Pl}} / 8 \pi$, the cosmological constant $\Lambda$, scalar squared masses $m_{a b}^{2}$, scalar cubics $A_{a b c}$, fermion masses $M_{i j}$ defined as

$$
\begin{aligned}
\mathscr{L}_{\text {massive }}= & -\frac{1}{2} \bar{M}_{\mathrm{Pl}}^{2} R-\Lambda-\frac{1}{2} m_{a b}^{2} \phi_{a} \phi_{b} \\
& -\frac{1}{6} A_{a b c} \phi_{a} \phi_{b} \phi_{c}-\frac{1}{2}\left(M_{i j} \psi_{i} \psi_{j}+\text { h.c. }\right) .
\end{aligned}
$$

The RGE for the massive terms can be obtained from the generic dimensionless RGE by considering one neutral scalar $s$ as a dummy non-dynamical variable, such that

$$
\begin{aligned}
& \bar{M}_{\mathrm{Pl}}^{2}=\xi_{s s} s^{2}, \quad \Lambda=\lambda_{s s s s} \frac{s^{4}}{4 !}, \quad M_{i j}=Y_{i j}^{s} s, \\
& m_{a b}^{2}=\lambda_{a b s s} \frac{s^{2}}{2}, \quad A_{a b c}=\lambda_{a b c s} s .
\end{aligned}
$$

This technique has been used to determine the RGE of massive parameters in generic QFT without gravity [38]. Gravitational couplings have been included in some less general models in $[27,73]$. The generic RGE of massive parameters in agravity are

$$
\begin{aligned}
(4 \pi)^{2} \frac{\mathrm{d} \bar{M}_{\mathrm{Pl}}^{2}}{\mathrm{~d} \ln \bar{\mu}}= & \frac{1}{3} m_{a a}^{2}+\frac{1}{3} \operatorname{Tr}\left(M^{\dagger} M\right)+2 \xi_{a b} m_{a b}^{2} \\
& +\left(\frac{2 f_{0}^{2}}{3}-\frac{5 f_{2}^{4}}{3 f_{0}^{4}}+2 X\right) \bar{M}_{\mathrm{Pl}}^{2}, \\
(4 \pi)^{2} \frac{\mathrm{d} \Lambda}{\mathrm{d} \ln \bar{\mu}}= & \frac{m_{a b}^{2} m_{a b}^{2}}{2}-\operatorname{Tr}\left[\left(M M^{\dagger}\right)^{2}\right]+\frac{5 f_{2}^{4}+f_{0}^{4}}{8} \bar{M}_{\mathrm{Pl}}^{4} \\
& +\left(5 f_{2}^{2}+f_{0}^{2}\right) \Lambda+4 \Lambda X \\
(4 \pi)^{2} \frac{\mathrm{d} M}{\mathrm{~d} \ln \bar{\mu}}= & \frac{1}{2}\left(Y^{\dagger b} Y^{b} M+M Y^{\dagger b} Y^{b}\right) \\
& +2 Y^{b} M^{\dagger} Y^{b}+Y^{b} \operatorname{Tr}\left(Y^{\dagger b} M\right) \\
& -3\left\{C_{2 F}, M\right\}+\frac{15}{8} f_{2}^{2} M+M X \\
(4 \pi)^{2} \frac{\mathrm{d} m_{a b}^{2}}{\mathrm{~d} \ln \bar{\mu}}= & \lambda_{a b e f} m_{e f}^{2}+A_{a e f} A_{b e f}-2\left[\operatorname{Tr}\left(Y^{\{a} Y^{\dagger b\}} M M^{\dagger}\right)\right. \\
& +\operatorname{Tr}\left(Y^{\dagger\{a} Y^{b\}} M^{\dagger} M\right)+\operatorname{Tr}\left(Y^{a} M^{\dagger} Y^{b} M^{\dagger}\right) \\
& \left.+\operatorname{Tr}\left(M Y^{\dagger a} M Y^{\dagger b}\right)\right] \\
& +\frac{5}{2} f_{2}^{4} \xi_{a b} \bar{M}_{\mathrm{Pl}}^{2}+\frac{f_{0}^{4}}{2}\left(\xi_{a b}+6 \xi_{a e} \xi_{e b}\right) \bar{M}_{\mathrm{Pl}}^{2} \\
& +f_{0}^{2}\left(m_{a b}^{2}+3 \xi_{b f} m_{a f}^{2}+3 \xi_{a f} m_{b f}^{2}+6 \xi_{a e} \xi_{b f} m_{e f}^{2}\right) \\
& +m_{a b}^{2}\left[\sum_{k=a, b}\left(Y_{2}^{k}-3 C_{2}^{k}\right)+5 f_{2}^{2}+2 X\right]
\end{aligned}
$$

$$
\begin{aligned}
(4 \pi)^{2} \frac{\mathrm{d} A_{a b c}}{\mathrm{~d} \ln \bar{\mu}}= & \lambda_{a b e f} A_{e f c}+\lambda_{a c e f} A_{e f b}+\lambda_{b c e f} A_{e f a} \\
& -2 \operatorname{Tr}\left(Y^{\{a} Y^{\dagger b} Y^{c\}} M^{\dagger}\right)-2 \operatorname{Tr}\left(Y^{\dagger\{c} Y^{a} Y^{\dagger b\}} M\right) \\
& +f_{0}^{2}\left(A_{a b c}+3 \xi_{a f} A_{f b c}+3 \xi_{b f} A_{f a c}+3 \xi_{c f} A_{f a b}\right) \\
& +6 f_{0}^{2}\left(\xi_{a e} \xi_{b f} A_{e f c}+\xi_{a e} \xi_{c f} A_{e f b}+\xi_{b e} \xi_{c f} A_{e f a}\right) \\
& +A_{a b c}\left[\sum_{k=a, b, c}\left(Y_{2}^{k}-3 C_{2}^{k}\right)+5 f_{2}^{2}+X\right],
\end{aligned}
$$

where the curly brackets represent the sum over the permutations of the corresponding indices: e.g. $Y^{\{a} Y^{\dagger b}=$ $Y^{a} Y^{\dagger b}+Y^{b} Y^{\dagger a}$. Notice that $X$ is a gauge-dependent quantity, equal to

$X=\frac{\left(3 c_{g}^{2}-12 c_{g}+13\right) \xi_{g}}{4\left(c_{g}-2\right)}+\frac{3\left(c_{g}-1\right)^{2} f_{0}^{2}}{4\left(c_{g}-2\right)^{2}}$

using the gauge-fixing action of [4], which depends on two free parameters $\xi_{g}$ and $c_{g}$ :

$$
\begin{aligned}
S_{\mathrm{gf}} & =-\frac{1}{2 \xi_{g}} \int \mathrm{d}^{4} x f_{\mu} \partial^{2} f_{\mu}, \\
f_{\mu} & =\partial_{\nu}\left(h_{\mu \nu}-c_{g} \frac{1}{2} \eta_{\mu \nu} h_{\alpha \alpha}\right) .
\end{aligned}
$$

The RGE of massive parameters are gauge dependent because the unit of mass is gauge dependent. Any dimensionless ratio of dimensionful parameters is physical and the corresponding RGE is gauge-independent, as can easily be checked from Eqs. (54a)-(54e). For example [4] gave the RG equation for $M_{h}^{2} / \bar{M}_{\mathrm{Pl}}^{2}$.

\section{References}

1. R. Utiyama, B.S. DeWitt, Renormalization of a classical gravitational field interacting with quantized matter fields. J. Math. Phys. 3, 608 (1962). [InSpires:Utiyama:1962sn]

2. S. Weinberg, Problems in gauge field theories. [InSpires:Weinberg:1974tw]

3. K.S. Stelle, Renormalization of higher derivative quantum gravity. Phys. Rev. D 16, 953 (1977). [In- Spires:Stelle:1976gc]

4. A. Salvio, A. Strumia, Agravity. JHEP 1406, 080 (2014). [arXiv:1403.4226]

5. C. Wetterich, Inflation, quintessence, and the origin of mass. Nucl. Phys. B897, 111 (2015). [arXiv:1408.0156]

6. K. Kannike, G. Hutsi, L. Pizza, A. Racioppi, M. Raidal, A. Salvio, A. Strumia, Dynamically induced planck scale and inflation. JHEP 1505, 065 (2015). [arXiv:1502.01334]

7. K. Kannike, A. Racioppi, M. Raidal, Linear inflation from quartic potential. JHEP 1601, 035 (2016). [arXiv:1509.05423]

8. A. Farzinnia, S. Kouwn, Classically scale invariant inflation, supermassive WIMPs, and adimensional gravity. Phys. Rev. D 93, 063528 (2016). [arXiv:1512.05890]

9. A. Salvio, Inflationary perturbations in no-scale theories. Eur. Phys. J. C 77, 267 (2017). [arXiv:1703.08012]

10. F.d O. Salles, I.L. Shapiro, Do we have unitary and (super)renormalizable quantum gravity below the Planck scale? Phys. Rev. D 89, 084054 (2014). [arXiv:1401.4583] 
11. M.M. Ivanov, A.A. Tokareva, Cosmology with a light ghost. JCAP 1612, 018 (2016). [arXiv:1610.05330]

12. T. Biswas, A. Mazumdar, W. Siegel, Bouncing universes in stringinspired gravity. JCAP 0603, 009 (2006). [arXiv:hep-th/0508194]

13. M. Ostrogradski, Memoire sur les equations differentielles relatives au probleme des isoperimetres. Mem. Acc. St. Petersbourg VI, 385 (1850)

14. A. Salvio, A. Strumia, Quantum mechanics of 4-derivative theories. Eur. Phys. J. C 76, 227 (2016). [arXiv:1512.01237]

15. M. Raidal, H. Veermäe, On the Quantisation of Complex Higher Derivative Theories and Avoiding the Ostrogradsky Ghost. Nucl. Phys. B 916, 607-626 (2017-03). [arXiv:1611.03498]

16. B. Holdom, J. Ren, QCD analogy for quantum gravity. Phys. Rev. D 93, 124030 (2016). [arXiv:1512.05305]

17. A. Salvio, Solving the standard model problems in softened gravity. Phys. Rev. D 94, 096007 (2016). [arXiv:1608.01194]

18. D. Anselmi, M. PivaA new formulation of Lee-Wick quantum field theory. JHEP 1706, 066 (2017). [arXiv:1703.04584]

19. D. Anselmi, M. PivaPerturbative unitarity of Lee-Wick quantum field theory. Phys. Rev. D 96(4), 045009 (2017). arXiv: 1703.05563

20. J.F. Donoghue, Quartic propagators, negative norms and the physical spectrum. Phys. Rev. D 96(4), 044007 (2017). arXiv: 1704.01533

21. G. Narain, Signs and stability in higher-derivative gravity. [arXiv:1704.05031]

22. D. Anselmi, On the quantum field theory of the gravitational interactions. JHEP 1706, 086 (2017). arXiv:1704.07728

23. G.F. Giudice, G. Isidori, A. Salvio, A. Strumia, Softened gravity and the extension of the standard model up to infinite energy. JHEP 1502, 137 (2015). [arXiv:1412.2769]

24. G.M. Pelaggi, A. Strumia, S. Vignali, Totally asymptotically free trinification. JHEP 1508, 130 (2015). [arXiv:1507.06848]

25. G.M. Pelaggi, F. Sannino, A. Strumia, E. Vigiani, Naturalness of asymptotically safe Higgs. [arXiv:1701.01453]

26. I.G. Avramidi, A.O. Barvinsky, Asymptotic freedom in higher derivative quantum gravity. Phys. Lett. B 159, 269 (1985). https:// doi.org/10.1016/0370-2693(85)90248-5

27. I.G. AvramidiCovariant methods for the calculation of the effective action in quantum field theory and investigation of higher derivative quantum gravity. [arXiv:hep-th/9510140]

28. G. de Berredo-Peixoto, I.L. Shapiro, Higher derivative quantum gravity with Gauss-Bonnet term. Phys. Rev. D 71, 064005 (2005). [arXiv:hep-th/0412249]

29. J. Julve, M. Tonin, Quantum gravity with higher derivative terms. Nuovo Cim. B 46, 137 (1978). https://doi.org/10.1007/ BF02748637

30. E.S. Fradkin, A.A. Tseytlin, Renormalizable asymptotically free quantum theory of gravity. Nucl. Phys. B 201, 469 (1982). https:// doi.org/10.1016/0550-3213(82)90444-8

31. M.B. Einhorn, D.R.T. Jones, Induced gravity I: real scalar field. JHEP 1601, 019 (2016). [arXiv:1511.01481]

32. M.B. Einhorn, D.R.T. Jones, Induced gravity II: grand unification. JHEP 1605, 185 (2016). [arXiv:1602.06290]

33. G. 't Hooft, The conformal constraint in canonical quantum gravity. [arXiv:1011.0061]

34. G. 't Hooft, A class of elementary particle models without any adjustable real parameters. Found. Phys 41, 1829 (2011). [arXiv:1104.4543]

35. G. 't Hooft, Singularities, horizons, firewalls, and local conformal symmetry. [arXiv: 1511.04427]

36. K. Hamada, Resummation and higher order renormalization in 4-D quantum gravity. Prog. Theor. Phys. 108, 399 (2002). [InSpires:Hamada:2002cm]

37. K. Hamada, Renormalizable 4D quantum gravity as a perturbed theory from CFT. Found. Phys. 39, 1356 (2009). [arXiv:0907.3969]
38. M.X. Luo, H.W. Wang, Y. Xiao, Two loop renormalization group equations in general gauge field theories. Phys. Rev. D 67, 065019 (2003). [arXiv:hep-ph/0211440]

39. P.D. Mannheim, Comprehensive solution to the cosmological constant, zero-point energy, and quantum gravity problems. Gen. Relat. Grav. 43, 703 (2009). [arXiv:0909.0212]

40. P.D. Mannheim, Making the case for conformal gravity. Found. Phys. 42, 388 (2011). [arXiv:1101.2186]

41. E.S. Fradkin, A.A. Tseytlin, Renormalizable asymptotically free quantum theory of gravity. Nucl. Phys. B 201, 469 (1982). https:// doi.org/10.1016/0550-3213(82)90444-8

42. I. Antoniadis, P.O. Mazur, E. Mottola, Conformal symmetry and central charges in four-dimensions. Nucl. Phys. B 388, 627 (1992). [arXiv:hep-th/9205015]

43. I.L. Shapiro, A.G. Zheksenaev, Gauge dependence in higher derivative quantum gravity and the conformal anomaly problem. Phys. Lett. B 324, 286 (1994). https://doi.org/10.1016/ 0370-2693(94)90195-3

44. G. de Berredo-Peixoto, I.L. Shapiro, Conformal quantum gravity with the Gauss-Bonnet term. Phys. Rev. D 70, 044024 (2003). [arXiv:hep-th/0307030]

45. G. Narain, R. Anishetty, Charge renormalization due to graviton loops. JHEP 1307, 106 (2013). [arXiv:1211.5040]

46. G. Narain, R. Anishetty, Running couplings in quantum theory of gravity coupled with gauge fields. JHEP 1310, 203 (2013). [arXiv:1309.0473]

47. L.S. Brown, J.C. Collins, Dimensional renormalization of scalar field theory in curved space-time. Ann. Phys. 130, 215 (1980). https://doi.org/10.1016/0003-4916(80)90232-8

48. S.J. Hathrell, Trace anomalies and $\lambda \phi^{4}$ theory in curved space. Ann. Phys. 139, 136 (1982). https://doi.org/10.1016/ 0003-4916(82)90008-2

49. S.J. Hathrell, Trace anomalies and QED in curved space. Ann. Phys. 142, 34 (1982). https://doi.org/10.1016/0003-4916(82)90227-5

50. M.D. Freeman, The renormalization of nonabelian gauge theories in curved space-time. Ann. Phys. 153, 339 (1984). https://doi.org/ 10.1016/0003-4916(84)90022-8

51. I. Jack, H. Osborn, Analogs for the $c$ theorem for four-dimensional renormalizable field theories. Nucl. Phys. B 343, 647 (1990). https://doi.org/10.1016/0550-3213(90)90584-Z

52. O.V. Tarasov, A.A. Vladimirov, A.Y.Zharkov, The Gell-Mann-Low function of QCD in the three loop approximation. Phys. Lett. B 93, 429 (1980). https://doi.org/10.1016/0370-2693(80)90358-5

53. J. Frohlich, On the triviality of $\lambda \phi^{4}$ in $D$ dimensions theories and the approach to the critical point in $D \geq 4$ dimensions. Nucl. Phys. B 200, 281 (1982). [InSpires:Frohlich:1982tw]

54. M. Luscher, P. Weisz, Scaling laws and triviality bounds in the Lattice $\phi^{4}$ theory. 1. One component model in the symmetric phase. Nucl. Phys. B290, 25 (1987). [InSpires:Luscher:1987ay]

55. U. Wolff, Precision check on triviality of $\phi^{4}$ theory by a new simulation method. Phys. Rev. D 79, 105002 (2009). [arXiv:0902.3100]

56. C. Wetterich, Exact evolution equation for the effective potential. Phys. Lett. B 301, 90 (1993). https://doi.org/10.1016/ 0370-2693(93)90726-X

57. T. Papenbrock, C. Wetterich, Two loop results from one-loop computations and nonperturbative solutions of exact evolution equations. Z. Phys. C 65, 519 (1994). [arXiv:hep-th/9403164]

58. J. Berges, N. Tetradis, C. Wetterich, Nonperturbative renormalization flow in quantum field theory and statistical physics. Phys. Rept. 363, 223 (2000). [arXiv:hep-ph/0005122]

59. A. Nicolis, R. Rattazzi, E. Trincherini, The Galileon as a local modification of gravity. Phys. Rev. D 79, 064036 (2008). [arXiv:0811.2197]

60. I. Antoniadis, E. Mottola, 4-D quantum gravity in the conformal sector. Phys. Rev. D 45, 2013 (1992). https://doi.org/10.1103/ PhysRevD.45.2013 
61. I. Antoniadis, P.O. Mazur, E. Mottola, Cosmological dark energy: Prospects for a dynamical theory. New J. Phys. 9, 11 (2006). [arXiv:gr-qc/0612068]

62. M. Maggiore, Nonlocal infrared modifications of gravity. A review. Fundam. Theor. Phys. 187, 221 (2017). [arXiv:1606.08784]

63. Sakharov's induced gravity, A Modern perspective. K. Akama, Y. Chikashige, T. Matsuki and H. Terazawa, "Gravity and Electromagnetism as Collective Phenomena: a Derivation of Einstein's General Relativity". Prog. Theor. Phys. 60, 868-877 (1978)

64. S.L. Adler, A formula for the induced gravitational constant. Phys. Lett. B 95, 241 (1980)

65. A. Zee, Spontaneously generated gravity. Phys. Rev. D 23, 858 (1981)

66. N.N. Khuri, An upper bound for induced gravitation. Phys. Rev. Lett. 49, 513 (1982)

67. N.N. Khuri, The sign of the induced gravitational constant. Phys. Rev. D 26, 2664 (1982)
68. S. L. Adler, "Einstein Gravity as a Symmetry Breaking Effect in Quantum Field Theory", Rev. Mod. Phys. 54 (1982) 729 [Rev. Mod. Phys. 55 (1983) 837]

69. N. Arkani-Hamed, H. Georgi, M.D. Schwartz, Effective field theory for massive gravitons and gravity in theory space. Ann. Phys. 305, 96 (2002). [arXiv:hep-th/0210184]

70. G. Veneziano, S. Yankielowicz, An effective lagrangian for the pure $\mathrm{N}=1$ supersymmetric Yang-Mills theory. Phys. Lett. 113B, 231 (1982). https://doi.org/10.1016/0370-2693(82)90828-0

71. N.M. Davies, T.J. Hollowood, V.V. Khoze, M.P. Mattis, Gluino condensate and magnetic monopoles in supersymmetric gluodynamics. Nucl. Phys. B 559, 123 (1999). [arXiv:hep-th/9905015]

72. F. Sannino, M. Shifman, Effective Lagrangians for orientifold theories. Phys. Rev. D 69, 125004 (2003). [arXiv:hep-th/0309252]

73. I.L. Shapiro, J. Sola, Scaling behavior of the cosmological constant: interface between quantum field theory and cosmology. JHEP 0202, 006 (2000). [arXiv:hep-th/0012227] 